\title{
THE BRANCH POINT STRUCTURE OF EXTENSIONS OF INTERIOR BOUNDARIES
}

\author{
BY \\ MORRIS L. MARX
}

1. Introduction. Let $D \subset E^{2}$ be an open set such that $\bar{D}$ is compact and Boundary $D$ is an oriented Jordan curve. A mapping $f: \bar{D} \rightarrow E^{2}$ is said to be properly interior if $f$ is light, open, and sense-preserving on $D$, and a local homeomorphism (relative to $\bar{D}$ ) at each point of Boundary $D$. A theorem of Stoilow [6, Theorem 4.5, p. 98] states that if $f$ is properly interior, then at each point $p$ of $D$ there exists a closed two-cell neighborhood $N$ of $p$ on which $f$ is topologically equivalent to $w=z^{n}$ on $z \leqq 1$ for some positive integer $n$. The integer $n-1$ is called the multiplicity of $f$ at $p$. A branch point is a point at which the multiplicity is strictly larger than zero. Suppose $\delta$ : Boundary $D \rightarrow E^{2}$ is continuous; then $\delta$ is an interior boundary if there exists a properly interior $f: \bar{D} \rightarrow E^{2}$ such that $f \mid$ Boundary $D=\delta$. The mapping $f$ is said to be an extension of $\delta$ to $D$. If $\delta$ is an interior boundary and $f$ is any properly interior extension of $\delta$, then $f$ has only finitely many branch points, say $z_{1}, \ldots, z_{r}$ with multiplicities $\mu(1), \ldots, \mu(r)$. It is well known that $1+\mu(1)+\cdots+\mu(r)$ is always equal to the tangential winding number of $\delta$ [2, Theorem 20.1, p. 71]. What has not been considered is what minimal value $r$ can take for a given interior boundary and what multiplicities can occur for the minimal $r$. This is part of the problem of determining more about the branch points, poles, and zeroes of light open mappings of the two-cell into the two sphere (see [2, p. 76]). An algorithm is presented here which answers this question for the case that $\delta$ is a representation of a normal curve. A mapping $\delta$ of an oriented one-dimensional manifold $J$ into the complex plane $E^{2}$ is called a representation; if $\delta$ possesses a continuous nonvanishing tangent $\delta^{\prime}$ then $\delta$ is called a regular representation. An image point $\delta_{0}$ of a regular representation $\delta$ is a vertex if there exist exactly two distinct points $x$ and $y$ such that $\delta(x)=\delta(y)=\delta_{0}$ and if the tangents $\delta^{\prime}(x)$ and $\delta^{\prime}(y)$ are linearly independent. A regular representation is normal if it has a finite number of vertices and every other image point has but one pre-image. Two representations (regular representations) $\delta$ and $\varepsilon$ are equivalent if there exists a sense-preserving homeomorphism $\phi: J \rightarrow J$ such that $\varepsilon=\delta \circ \phi$ (and $\phi^{\prime}$ is continuous and nonvanishing). A regular (normal) curve is then defined to be an oriented curve with a regular (normal) representation.

In [4] Titus has given an algorithm to determine if a normal representation $\delta$ is an interior boundary. His method is to "cut" $\delta$ into two normal representations $\delta$ * and $\delta^{* *}$ in such a way that $\delta$ is an interior boundary if and only if $\delta^{*}$ and $\delta^{* *}$ are

Presented to the Society, August 31, 1967; received by the editors December 16, 1966. 
and $\delta^{*}$ and $\delta^{* *}$ have less vertices than $\delta$. The algorithm presented in this paper is closely related to Titus'; in fact, his cut of Type II' is unchanged here. The Titus cut of Type I destroys information about branch point structure and so another type of cut is introduced. This new cut algorithm not only answers questions about the branch point structure but can also be used to determine if a normal representation is an interior boundary. The price paid for this dual capability is increased complexity of the algorithm.

2. Preliminaries. In what follows $\delta$ will be a representation of a closed curve. Let $[\delta]$ denote the point set consisting of image points of $\delta$. Let $w(\delta, p)$ be the index of $\delta$ about a point $p$ not in [ $\delta]$.

The outer boundary of $\delta$ will be the subset of [ $\delta]$ which is contained in the closure of the unbounded component of the complement of $[\delta]$; an outer point $p$ is a point on the outer boundary such that $p$ has but one pre-image. For normal $\delta$ and any nonvertex $p$ one can define $w^{+}(\delta, p)$ and $w^{-}(\delta, p)$ as the larger and smaller winding numbers of points $p^{\prime}$ near $p$ but not on [ $\delta$ ]. An outer point $p$ is positive [negative] if $w^{+}(\delta, p)=1\left[w^{-}(\delta, p)=-1\right]$.

Let $\delta$ be normal and let $\delta(0)$ be an outer point where $\delta$ is given by the complexvalued function $\delta(t)=a(t)+i b(t)$ with $t$ the usual angle parameter, $0 \leqq t<2 \pi$. Index the $n$ vertices in the natural way by traversing the curve with increasing $t$ and using consecutively the integers $1,2, \ldots, n$; thus $\delta_{1}, \delta_{2}, \ldots, \delta_{n}$. (See Figure 1.)

The $2 n$ pre-images of the vertices will be denoted by the lower case Roman equivalent of the Greek letter denoting the curve and they will be indexed so that $0<d_{1}<\cdots<d_{2 n}<2 \pi$. Denote $d_{j}$ also by $d_{k}^{*}$ if $\delta\left(d_{j}\right)=\delta\left(d_{k}\right)$ for $j \neq k$; thus, $\delta\left(d_{k}^{*}\right)=\delta\left(d_{k}\right)$ for all $k$. Define the function $\nu$ by

$$
\nu\left(d_{k}\right)=\nu(k)=\operatorname{sgn}\left|\begin{array}{ll}
a^{\prime}\left(d_{k}^{*}\right) & b^{\prime}\left(d_{k}^{*}\right) \\
a^{\prime}\left(d_{k}\right) & b^{\prime}\left(d_{k}\right)
\end{array}\right| .
$$

Let $t_{k}^{\prime}$ yet be another name for the smaller pre-image of $\delta_{k}$ and let $t_{k}^{\prime \prime}$ be the other pre-image. Define the function $\lambda$ on the set of vertices by

$$
\lambda\left(\delta_{k}\right)=\lambda(k)=\operatorname{sgn}\left|\begin{array}{ll}
a^{\prime}\left(t_{k}^{\prime \prime}\right) & b^{\prime}\left(t_{k}^{\prime \prime}\right) \\
a^{\prime}\left(t_{k}^{\prime}\right) & b^{\prime}\left(t_{k}^{\prime}\right)
\end{array}\right| .
$$

Given any pair of distinct vertices $\delta_{j}$ and $\delta_{k}$, define the following relations:

(1) $j \supset k$ if $t_{j}^{\prime}<t_{k}^{\prime}<t_{k}^{\prime \prime}<t_{j}^{\prime \prime}$.

(2) $j R k$ if $t_{k}^{\prime}<t_{j}^{\prime}<t_{k}^{\prime \prime}<t_{j}^{\prime \prime}$.

(3) $j L k$ if $k R j$.

(4) $j \mid k$ if either $t_{j}^{\prime}<t_{j}^{\prime \prime}<t_{k}^{\prime}<t_{k}^{\prime \prime}$ or $t_{k}^{\prime}<t_{k}^{\prime \prime}<t_{j}^{\prime}<t_{j}^{\prime \prime}$.

For a normal $\delta$ with $p=\delta(0)$ a positive outer point, the intersection sequence of $\delta$ with respect to $p$ is defined by the sequence $\left\{d_{k}\right\}$, the values of $d_{k}^{*}$, and the values of $\nu(k)$ for each $k$. A pair of normal representations $\delta$ and $\nu$ have isomorphic intersection sequences if they have the same number of vertices, $d_{j}^{*}=d_{k}$ if $e_{j}^{*}=e_{k}$, and $v\left(d_{k}\right)=v\left(e_{k}\right)$. As will be seen from the following theorem if two representations 


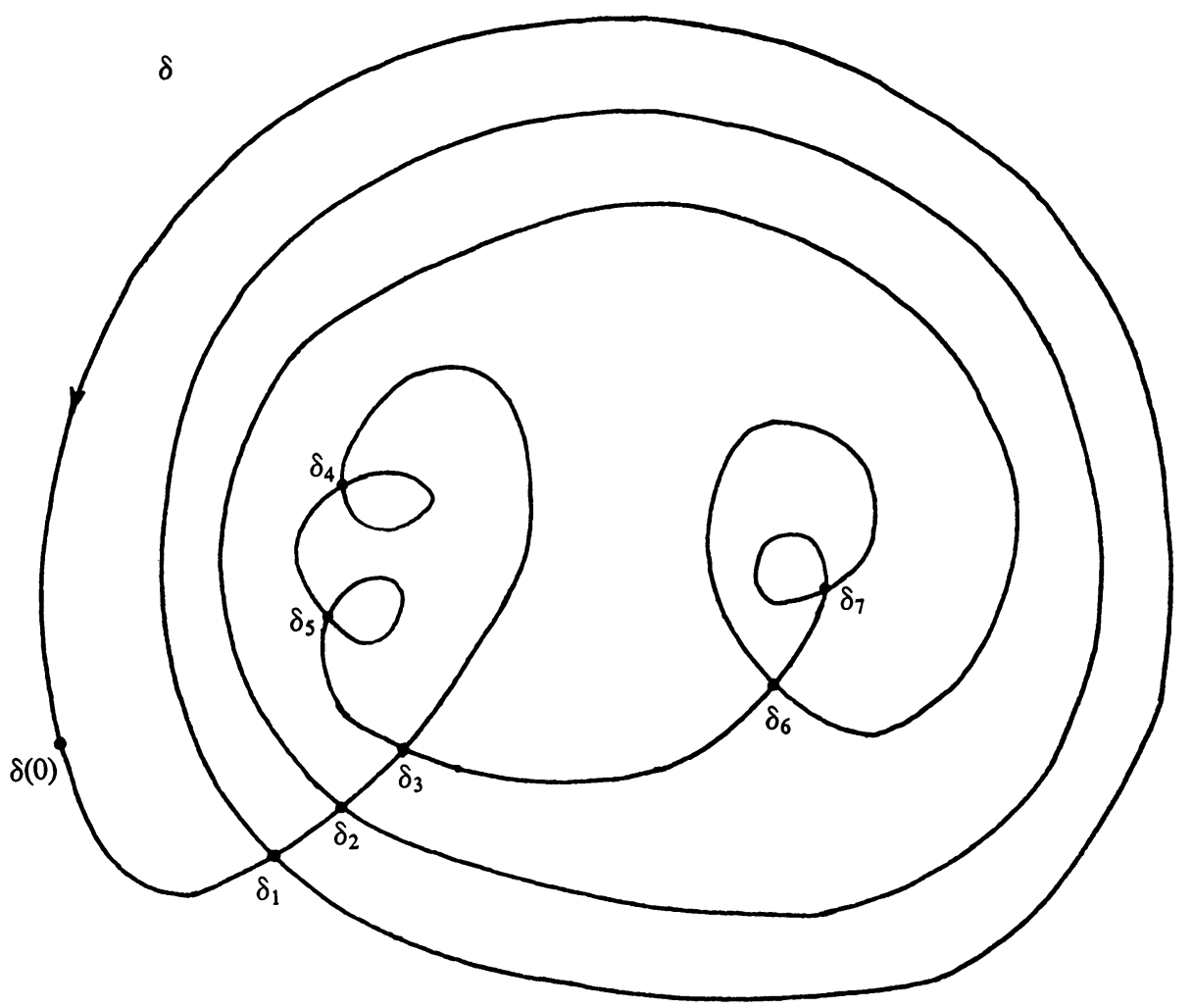

FIGURE 1

have isomorphic intersection sequences, they are equivalent; moreover, if one has an extension to the disk with certain topological properties, so will the other. Hence we consider two representations with isomorphic intersection sequences as interchangeable.

THEOREM [4, p. 49]. Let $\delta$ be a normal representation defined on a Jordan curve $D$; let $\varepsilon$ be normal and defined on the Jordan curve E. Suppose that each curve has a positive outer point and the curves have isomorphic intersection sequences. Then there exists a sense-preserving homeomorphism $h$ of $E^{2}$ onto $E^{2}$ taking $D$ onto $E$ such that $\varepsilon h=\delta$ (juxtaposition denotes function composition).

Suppose $\delta$ and $\varepsilon$ are representations and $x=\delta(p)$ and $y=\delta(q)$ are two points with $p<q$. Denote by $\delta(p) \delta(q)$ or $\delta(p) \delta(q)(\delta)$ the representation obtained by restricting $\delta$ to $p \leqq t \leqq q$. If $x$ and $y$ each have only one pre-image, then $x y$ or $x y(\delta)$ will also denote this representation. Let $-\delta$ represent the curve obtained by tracing $\delta$ in the opposite direction. Consistent with this notation $\delta(p) \delta(q)(-\delta)$ traces from $\delta(q)$ to $\delta(p)$ via $-\delta$. We shall use $a p(\delta)+p b(\varepsilon)$ to denote the representation $a p(\delta)$ followed continuously by $p b(\varepsilon)$, where $p$ is in $[\delta] \cap[\varepsilon]$.

If $J$ is a Jordan curve in $E^{2}$, then Ins $J$ denotes the bounded component of 
$E^{2}-J$. The set Ins $J$ will be called a Jordan domain. If $a$ and $b$ are two points of $J$, then $[a, b]$ denotes the set of points encountered as $J$ is traversed from $a$ to $b$ in the positive orientation; also $(a, b)$ denotes $[a, b]-\{a, b\}$.

We will assume from this point on that a properly interior mapping $f$ on $\bar{D}$ does not map branch points onto $f(\bar{D}-D)$. There is no loss of generality in this assumption for if $f$ does not have this property, there exists a homeomorphism $h: D \rightarrow D$ such that $f$ composed with $h$ has the property. For our purposes $f$ and $f \circ h$ are interchangeable.

3. Representations with $\lambda \equiv 1$. Let $\delta$ be a normal representation with a properly interior extension $f$. Suppose $f$ has $r$ distinct branch points with multiplicities $\mu(1), \mu(2), \ldots, \mu(r)$. The sum $1+\mu(1)+\cdots+\mu(r)$ is always equal to the tangential winding number of $\delta$ [2, Theorem 20.1, p. 71]. There remains the question of what minimum value $r$ can take, and in that case, what can the multiplicities be. The results of this section answer that question for the class of representations $\delta$ such that $\lambda\left(\delta_{j}\right)=1$ for every vertex $\delta_{j}$ (for brevity we write $\lambda \equiv 1$ ). Implicit in the previous remark is the fact that a representation $\delta$ with $\lambda \equiv 1$ is an interior boundary. We shall state that as soon as we develop a few preliminaries.

Consider the vertices of $\delta$ as a partially ordered set under the relation $\delta_{j}>\delta_{k}$ if $j \supset k$. A chain will be a maximal totally ordered set of vertices. A normal $\delta$ is called properly nested if for any two vertices $\delta_{j}$ and $\delta_{k}$ either $j \supset k, k \supset j$, or $j \mid k$.

LEMMA 1. Suppose $\delta$ is a normal representation with a positive outer point. If $\lambda(k)=1$ for $k=1,2, \ldots, n$, then $\delta$ has nonnegative winding number at every point and $\delta$ is properly nested.

Proof. It follows from [3, Theorem 4, p. 1090] that $\delta$ has nonnegative winding number at every point.

Suppose by way of contradiction that $\delta$ is not properly nested. Then there exist $d_{j}$ and $d_{k}$ such that $d_{k}<d_{j}<d_{k^{*}}<d_{j^{*}}$, i.e., $j R k$. Suppose $k$ is the smallest integer such that there is a $j$ with $j R k$. Pick a point $u$ such that $d_{k-1}<u<d_{k}$ and let $T$ be the interval from $d_{k}$ to $d_{k^{*}}$. In this case $\delta(u)$ is in the unbounded component of the complement of $[\delta \mid T]$. Otherwise, there would be a point $d_{m}$ such that $d_{m}<d_{k}<$ $d_{m^{*}}<d_{k^{*}}$, which contradicts the minimality of $k$. Now Corollary 1 to Theorem 1 of $[3$, p. 1087] applies; by this result, the sum over all $\lambda(j)$ with $j R k$ is zero. This contradicts the fact that $\lambda \equiv 1$.

Definition. Suppose $\delta_{m}$ is a vertex; then $t_{m}^{\prime \prime}=d_{r}$ for some $r$. We say $\delta_{m}$ is a terminal vertex if $\lambda(m)=1$ and $t_{m}^{\prime}=d_{r-1}$. If $T$ is the interval $t_{m}^{\prime} \leqq t \leqq t_{m}^{\prime \prime}$, then $\delta^{*}=\delta \mid T$ represents a positively oriented Jordan curve and $\left[\delta^{*}\right] \cap[\delta]=\left\{\delta_{m}\right\}$.

Lemma 2. Suppose $\delta$ is as in Lemma 1 . Then $\delta_{m}$ is the smallest vertex in a chain of vertices if and only if $\delta_{m}$ is a terminal vertex.

Proof. Suppose $\delta_{m}$ is the smallest vertex in a chain and let $t_{m}^{\prime \prime}=d_{k}$. By hypothesis on $\delta, \lambda\left(\delta_{m}\right)=1$. To complete the proof, it suffices to show $t_{m}^{\prime}=d_{k}^{*}=d_{k-1}$. 
If $d_{k}^{*}$ is not $d_{k-1}$, then $d_{k}^{*}<d_{k-1}$; thus, one of the following holds:

(1) $d_{k-1}^{*}<d_{k}^{*}<d_{k-1}<d_{k}$,

(2) $d_{k}^{*}<d_{k-1}<d_{k}<d_{k-1}^{*}$

(3) $d_{k}^{*}<d_{k-1}^{*}<d_{k-1}<d_{k}$.

Cases (1) and (2) cannot hold, since by Lemma $1, \delta$ is properly nested. If (3) holds, then the vertex $\delta\left(d_{k-1}\right)=\delta_{p}$ is such that $m \supset p$; but $\delta_{m}$ is minimal. It must be then that $d_{k}^{*}=d_{k-1}$.

The converse follows immediately from the definition of terminal vertex.

THEOREM. If $\delta$ is a representation with $\lambda \equiv 1$, then $\delta$ is an interior boundary.

Proof. If $\delta_{m}$ is the smallest vertex in a chain, then it follows from Lemma 2 that $\delta$ has a cut of type I at $\delta_{m}$ and that $\delta^{*}$ is a representation with $\lambda \equiv 1$. (See Paragraph 7 of [4].) By Theorems 4 and 5 of [4] we have that $\delta$ is an interior boundary if and only if $\delta^{*}$ is. But $\delta^{*}$ has one less vertex than $\delta$ [4, Theorem 6, p. 55]. An induction argument completes the proof.

LEMMA 3. Let $\delta$ be a normal representation defined on the boundary of a Jordan domain $D$ and suppose $f$ is a properly interior extension of $\delta$ to $\bar{D}$. Let $\delta_{m}$ be a terminal vertex of $\delta$. If $T$ is the interval $t_{m}^{\prime} \leqq t \leqq t_{m}^{\prime \prime}$, then there exists a Jordan curve $J \subset \bar{D}$ such that $J \cap(\bar{D}-D)=T, f(J)=f(T)$, and for some $k>1, f \mid J$ is topologically equivalent to $w=z^{k}$ on $|z|=1$.

Proof. Pick $s$ in $T$ so that $t_{m}^{\prime}<s<t_{m}^{\prime \prime}$. Let $\gamma=\delta\left|\left[t_{m}^{\prime}, s\right], \eta=\delta\right|\left[s, t_{m}^{\prime \prime}\right]$, and $M=$ $[\gamma] \cup[\eta]$. Because $\lambda\left(\delta_{m}\right)=1$, Theorem 1 of [1, p. 49] applies and there is an arc $H_{1}$ in $\bar{D}$, with one end point at $t_{m}^{\prime}$, such that $H_{1}$ intersects $\bar{D}-D$ only at $t_{m}^{\prime}$. For the other end point $y_{1}, f\left(y_{1}\right)=\delta(s)$; also $f\left(H_{1}\right) \subset[\eta]$.

Applying this same theorem at $y_{1}$, we produce an arc $K_{1}$ with $y_{1}$ one end point, $f\left(K_{1}\right) \subset[\gamma]$, and $K_{1}$ intersects $\bar{D}-D$ only at its other end point $z_{1}$, if at all. Let $L_{1}=H_{1} \cup K_{1}$; note that $f\left(L_{1}\right) \subset M$. If $z_{1} \in D$, then $f\left(z_{1}\right)=\delta_{m}$ and a repetition of the above process produces an $\operatorname{arc} L_{2}$ with end point at $z_{1}$ such that $f\left(L_{2}\right) \subset M$. This process must terminate after a finite number of steps since properly interior mappings are finite-to-one. Thus, the last arc produced, say $L_{q}$, must have one end point in $\bar{D}-D$. Since $f$ is a local homeomorphism at each point of $\bar{D}-D$, this end point must be $t_{m}^{\prime \prime}$. Hence, the arc $P=L_{1} \cup \cdots \cup L_{q}$ intersects $\bar{D}-D$ only in the points $t_{m}^{\prime}$ and $t_{m}^{\prime \prime}$; also, $f(P)=M$. Then $J=P \cup T$ is the desired Jordan curve.

On $J$ the mapping $f$ is topologically equivalent to $w=z^{k}$ on $|z|=1$ for some $k>1$ by Theorem $4.3[6$, p. 86].

Definition. Let $\delta, f$, and $J$ be as in Lemma 3. Any branch points of $f$ (or their multiplicities) in Ins $J$ are said to belong to the terminal vertex $\delta_{m}$.

Let $\mu$ be a finite sequence of positive integers, i.e., $\mu=\mu(1), \ldots, \mu(k)$. Define $|\mu|=k$. We do not require that the $\mu(j)$ be distinct nor that $|\mu|$ be the same for every $\mu$ considered. For an interior boundary $\delta$, define $\Gamma(\delta)$ to be the set of all sequences $\mu$ such that $\delta$ has a properly interior extension $f$ with exactly $|\mu|$ branch 
points of multiplicities $\mu(1), \mu(2), \ldots, \mu(k)$. Note that for any $\mu \in \Gamma(\delta), \sum_{j=1}^{k} \mu(j)$ is equal to one plus the tangential winding number of $\delta$ [2, Theorem 20.1, p. 71]. It follows from this that $\delta$ has an extension which is a local homeomorphism if and only if the tangential winding number of $\delta$ is one; in this case, $\Gamma(\delta)$ is empty. We make the convention that $\Phi$ denotes the empty sequence. When $\delta$ has a local homeomorphism extension, we consider $\Phi$ the only element of $\Gamma(\delta)$. If $\mu$ and $\nu$ are any sequences as above, define $\mu \cup \nu$ to be the sequence $\mu(1), \ldots, \mu(k), \nu(1), \ldots$, $\nu(l)$. Define $\mu \cup \Phi=\mu$.

The following theorem gives an algorithm for constructing $\mu$ in $\Gamma(\delta)$ with $|\mu|$ minimal, where $\delta$ is a representation with $\lambda \equiv 1$. Before stating the theorem we make one definition.

Definition. Let $\delta$ be a representation with $\lambda \equiv 1$ where $\delta$ is defined on $[0,2 \pi]$. Suppose $\delta_{m}$ is the terminal vertex of a chain of $\delta$ of maximal length and assume this length is at least two. Define $I=\left\{\delta_{i} \mid p \supset i\right\}$ where $\delta_{p}$ is the smallest vertex in the chain such that $p \supset m$. Each $\delta_{i}$ in $I$ is a terminal vertex by the maximality of the length of the chain. Of course, $\delta_{m}$ is in $I$. For each $\delta_{i}$ in $I$ let $T_{i}=\left[t_{i}^{\prime}, t_{i}^{\prime \prime}\right]$. Define $\delta^{*}$ to be the representation given by $\delta \mid\left([0,2 \pi]-\bigcup\left\{T_{i} \mid \delta_{i}\right.\right.$ in $\left.\left.I\right\}\right)$; thus, $\delta^{*}$ is obtained by removing from $\delta$ the loops corresponding to the vertices $\delta_{i}$ in $I$. Suppose we renumber the vertices of $\delta$ so that $\delta_{1}, \ldots, \delta_{r}$ are the terminal vertices of $\delta$ not in $I$ and $\delta_{r+1}, \ldots, \delta_{s}$ the terminal vertices of $\delta$ in $I$. We number the vertices of $\delta^{*}$ so that $\delta_{j}^{*}$ is the terminal vertex of $\delta^{*}$ corresponding to $\delta_{j}$ (new numbering), $1 \leqq j \leqq r$, and $\delta_{r+1}^{*}$ corresponds to the vertex $\delta_{p}$ of $\delta$ in the old numbering. (See Figures 1 and 2.)

Suppose that $\delta$ has every chain of length 1 and has $s$ such chains. By Lemma 3, $\delta$ has at least $s$ branch points. But the tangential winding number of $\delta$ is $s+1$ and so every branch point has multiplicity one. We can thus ignore this case.

THEOREM 1. Suppose $\delta$ is a representation with $\lambda \equiv 1$ with vertices numbered as in the definition above.

(1) If $\mu$ is in $\Gamma(\delta)$, then $|\mu| \geqq s$, where $s$ is the number of terminal vertices of $\delta$. If $|\mu|=s$, then there is a 1-1 correspondence between the terminal vertices and the $\mu(j)$.

(2) Suppose $\mu$ is a sequence of positive integers with $|\mu|=s$. Then $\mu$ is in $\Gamma(\delta)$ with $\mu(j)$ belonging to the terminal vertex $\delta_{j}, 1 \leqq j \leqq s$, if and only if $\mu^{*}$ is in $\Gamma\left(\delta^{*}\right)$ where

$$
\begin{aligned}
\mu^{*}(j) & =\mu(j) \quad \text { for } 1 \leqq j \leqq r, \\
\mu^{*}(r+1) & =\sum_{j=r+1}^{s}(\mu(j)-1)
\end{aligned}
$$

with $\mu^{*}(j)$ belonging to $\delta_{j}^{*}, 1 \leqq j \leqq r+1$.

Proof. We first dispose of (1) by appealing to Lemma 3. To each terminal vertex there corresponds a Jordan curve $J$ on which $f$, an extension of $\delta$, is topologically equivalent to $w=z^{k}, k>1$, on $|z|=1$. There must be a branch point in Ins $J$; 


$$
\delta^{2}=\delta^{*}
$$

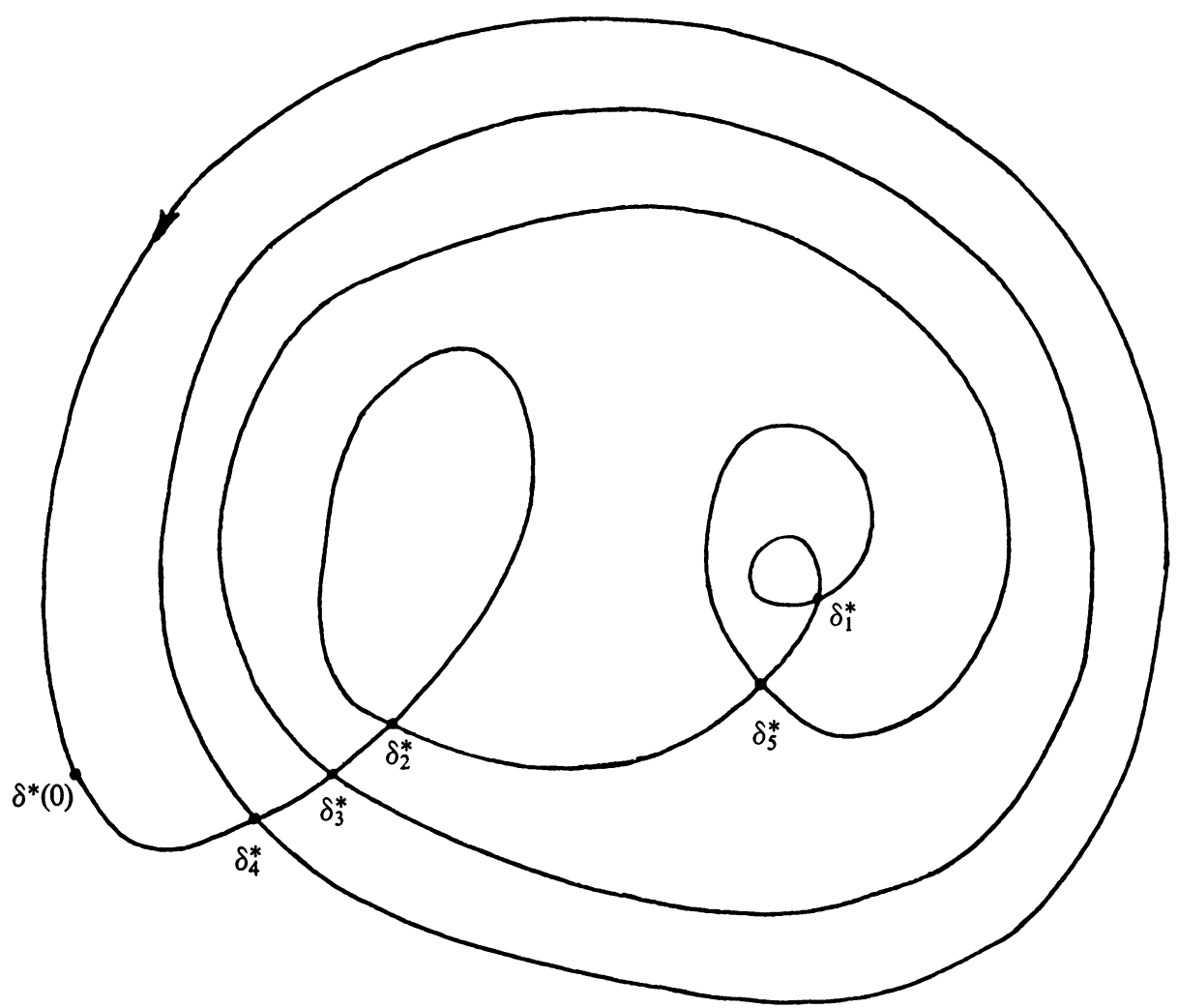

FIGURE 2

if $|\mu|$ is to be minimal there can be only one, since $f$ can be redefined to be topologically equivalent to $w=z^{k}$ on $|z|<1$ in Ins $J$.

Now we turn our attention to (2). First suppose $\mu$ is in $\Gamma(\delta)$ with $|\mu|$ minimal. Let $f$ be an extension of $\delta$ to $\bar{D}$ with branch point structure given by $\mu$, where $\mu(j)$ belongs to the terminal vertex $\delta_{j}, 1 \leqq j \leqq s$.

Let $\delta_{j}$ be a terminal vertex in $I$. Then there exists a Jordan curve $J$ as in Lemma 3 such that, on $J, f$ is topologically equivalent to $w=z^{k}$ on $|z|=1$. Note that $k=\mu(j)-1$ since $\mu(j)$ belongs to $\delta_{j}$. We may assume that, on $J \cup \operatorname{Ins} J, f$ is topologically equivalent to $w=z^{k}$ on $|z| \leqq 1$. Thus, there are $\operatorname{arcs} X$ and $Y$ in Ins $J$ such that $X$ has end point $t_{j}^{\prime}, Y$ has end point $t_{j}^{\prime \prime}, X$ and $Y$ intersect only at their other end point, and $f(X)=f(Y)$. Let $D_{1}$ be the disk bounded by $\bar{D}-D-\left[t_{j}^{\prime}, t_{j}^{\prime \prime}\right]$ and $X \cup Y$. There exists a map $h$ from $\bar{D}_{1}$ onto $|z| \leqq 1$ such that $h$ is a homeomorphism on $\bar{D}_{1}-X-Y$, on $X$, and on $Y$. Also $h(X)=h(Y)=\{z \mid z$ real, $0 \leqq z \leqq 1\}$ and, for $x$ in $X$ and $y$ in $Y, h(x)=h(y)$ if and only if $f(x)=f(y)$. Clearly $g=f h^{-1}$ is well defined, continuous, light everywhere, and open, except possibly on $h(X)$; therefore, $g$ is open on $h(X)$ [5, Theorem 9, p. 336]. The map $g$ is a properly interior extension of 
$\delta$ with the loop corresponding to $\delta_{j}$ removed. Note that $g$ has the same branch point structure as $f$ except that the branch point of multiplicity $\mu(j)$ has been replaced by one of multiplicity $\mu(j)-1$.

We continue this process, removing the loops associated with the vertices in $I$ and obtaining an extension $f^{*}$ of $\delta^{*}$ with multiplicities $\mu(1), \mu(2), \ldots, \mu(r)$, $\mu(r+1)-1, \ldots, \mu(s)-1$. Applying Lemma 3 to $\delta_{r+1}^{*}$ yields a Jordan curve $J$ such that $f^{*} \mid J$ is topologically $w=z^{k}$ on $|z|=1$. If $z_{j}$ is the branch point of $f$ of multiplicity $\mu(j)$ belonging to $\delta_{j}$ in $I$, then by the construction above we can assume that there is a $z_{j}^{\prime}$ which is a branch point of $f^{*}$ of multiplicity $\mu(j)-1$. Since $p \supset j$ and $\mu(j)$ belongs to $\delta_{j}$, we can also assume that $z_{j}^{\prime}$ is in Ins $J$. Thus, $k=\sum_{j=r+1}^{s}(\mu(j)-1)=$ $\mu^{*}(r+1)$. We can define $f^{*}$ to be topologically $z^{\mu^{*}(r+1)+1}$ on $J \cup$ Ins $J$. This process produces an extension of $\delta^{*}$ with branch point structure $\mu^{*}$.

Now suppose $\mu^{*}$ belongs to $\Gamma\left(\delta^{*}\right)$ and let $f^{*}$ be a properly interior extension of $\delta^{*}$ to $\bar{D}$ with branch point structure given by $\mu^{*}$, where each $\mu^{*}(j)$ belongs to a terminal vertex of $\delta^{*}$. Let $\delta_{r+1}^{*}$ be as before and let $J$ and $T$ be as in Lemma 3. Then $f^{*} \mid J$ is topologically $z^{\mu^{*}(r+1)+1}$ on $|z|=1$. Consider the following

Claim. Let $J$ be a Jordan curve and suppose $f \mid J$ is topologically equivalent to $w=z^{k}$ on $|z|=1$. Let $T$ be a closed arc in $J$ such that $f$ maps both end points into the same point, but is $1-1$ on the rest of $T$. For any integers $\rho(1), \rho(2), \ldots, \rho(u)$ such that $\sum_{i=1}^{u} \rho(i)=k-1$, there exists a properly interior map $g$ on $J \cup$ Ins $J$ such that $g \mid J=f$ and $g$ has branch points of multiplicities $\rho(1), \ldots, \rho(u)$ (consequently, only those multiplicities occur). Furthermore, if $t_{1}, t_{2}, \ldots, t_{v}$ are any points of $T$, there exist arcs $\gamma^{1}, \gamma^{2}, \ldots, \gamma^{u}$ in $J \cup$ Ins $J$ such that for each $j, \gamma^{j}$ intersects $J$ only at $t_{j}$, the other end point of $\gamma^{j}$ is the branch point of multiplicity $\rho(j), f$ is $1-1$ on $\gamma^{j}$, and $\gamma^{i} \cap \gamma^{j}=\varnothing$ if $i \neq j$. We make a slight abuse of language and extend the claim to the case where some of the $\rho(i)=0$; i.e., some of the end points of the $\gamma^{i}$ need not be branch points.

If the claim is true, we apply it for $f^{*}, J$ and $T$ as in the paragraph preceeding the claim. Then $k=\mu^{*}(r+1)+1$ and let $\rho(i)=\mu(i)-1, r+1 \leqq i \leqq s$. By hypothesis $k-1=\mu^{*}(r+1)=\sum_{i=r+1}^{s} \rho(i)$. Suppose without loss of generality that $\bar{D}$ is the unit disk in the complex plane and $\gamma^{r+1}=\{z \mid z$ real, $0 \leqq z \leqq 1\}$. We define a mapping $h$ on the unit disk as follows. On the part of the unit disk with $\operatorname{Im} z \geqq 0$ define $h$ to be $f^{*}\left(z^{2}\right)$. Let $K$ be a Jordan curve tangent to $\left[\delta^{*}\right]$ at $f^{*}\left(t_{1}\right)$ and such that $f^{*}\left(\gamma^{1}\right)$ is in $K \cup$ Ins $K$ but intersects $K$ only at $f^{*}\left(t_{1}\right)$. Define $h$ on $\left\{e^{i \theta} \mid \pi \leqq \theta \leqq 2 \pi\right\}$ so that it traces $K$ in a positive orientation as $\theta$ increases from $\pi$ to $2 \pi$ and $h\left(e^{i \pi}\right)=h\left(e^{i 2 \pi}\right)$ $=f^{*}\left(t_{1}\right)$. Then $h$ can be defined on $\{z|0 \leqq| z \mid \leqq 1, \pi<\arg z<2 \pi\}$ to be a homeomorphism and so that $h$ is continuous on the whole disk. By Theorem 9 [5, p. 336], $h$ will be properly interior. Note that $h$ has a branch point at zero of multiplicity $\mu(r+1)$ and elsewhere the multiplicities are the same as those of $f^{*}$.

If we repeat this process for each of the other values $i=r+2, \ldots, s$, we will have produced a representation that adds the loops corresponding to the terminal vertices of $\delta$ in $I$. We also obtain a properly interior extension of this representation 
which has branch point structure as in (2). Hence, we have proved the theorem if we establish the claim.

The claim will be proved by induction on $u$. If $u=1$, the claim is clear. Suppose it true for $u-1>1$. Without loss of generality take $J$ and $f(J)$ to be the unit circle. By the induction hypothesis there is a map $g$, and $\operatorname{arcs} \gamma^{1}, \ldots, \gamma^{u-1}$ which satisfy the claim with respect to $z^{\rho(1)+\cdots+\rho(u-1)+1}$ and $\rho(1), \ldots, \rho(u-1)$. Let $\varepsilon^{\prime}$ be an arc in Ins $g(J)$ with one end point at $g\left(t_{v}\right)$, which does not intersect any $g\left(\gamma^{j}\right)$. By Theorem 1 [1, p. 49], there is an $\operatorname{arc} \varepsilon$ in Ins $J$ with one end point at $t_{v}$, mapping homeomorphically onto $\varepsilon$. Note we are done if $\rho(u)=0$. Assume again without loss of generality that $t_{v}=1, \varepsilon=\{z \mid z$ real, $0 \leqq z \leqq 1\}$, and $g \mid \varepsilon=z^{\rho(u)+1}$. Define $f$ on $J \cup$ Ins $J$ by letting $f$ be $g\left(z^{\rho(u)+1}\right)$ on $\{z|0 \leqq| z \mid \leqq 1,0 \leqq \arg z \leqq 2 \pi /(\rho(u)+1)\}$ and $z^{\rho(u)+1}$ on the rest of the unit disk. Then $f$ has the required properties, where $\gamma^{u}$ is $\varepsilon$. This establishes the claim and hence, the theorem.

Using Theorem 1 we can test whether $\mu$ is in $\Gamma(\delta)$ or not, with $|\mu|$ minimal, by examining $\mu^{*}$. There is an improvement in our situation since $\delta^{*}$ has all chains no longer than those of $\delta$ and at least one strictly shorter. Thus, repeated application

$$
\delta^{3}=\left(\delta^{2}\right)^{*}
$$

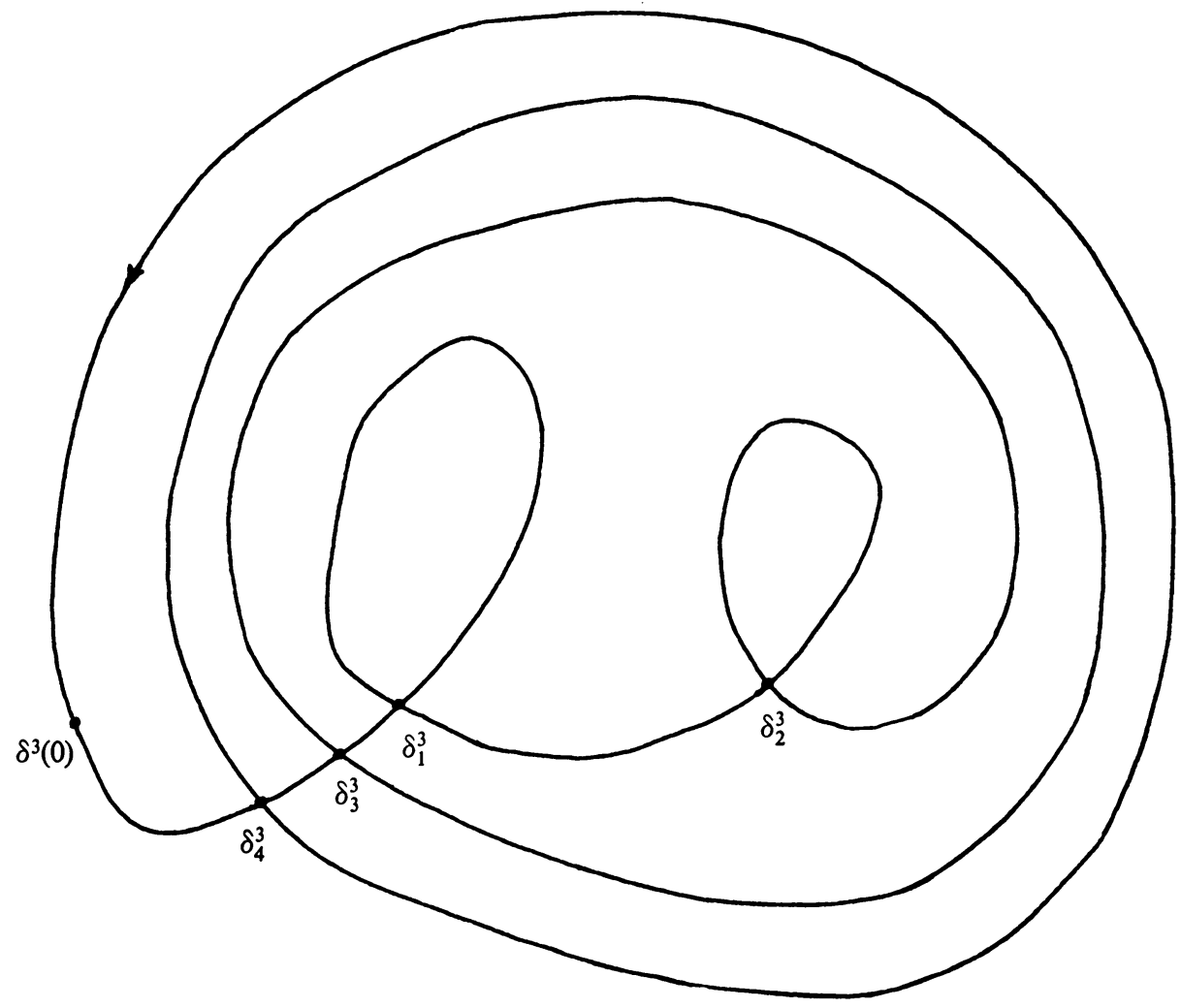

FIGURE 3 
of the theorem reduces the problem to examining a representation with every chain of length one. In this case the only admissible $\mu$ is one which has a branch point of multiplicity one belonging to each terminal vertex. Also, starting with such a representation we can build up to a $\mu$ in $\Gamma(\delta)$ by using the theorem in the other direction.

Note that at any point there may be several chains which are of maximal length and so there are several choices for which $\delta^{*}$ to use. Since the theorem is "if and only if" it is immaterial which $\delta^{*}$ is chosen.

EXAMPLE. In Figures $1-4$ we reduce a representation by the method of the theorem until we obtain the representation $\delta^{4}$ (Figure 4). It is clear that this representation has one branch point of multiplicity 2 . Working backwards we get

$\Gamma\left(\delta^{3}\right):(3,1) ;(2,2) ;(1,3)$. (The first entry belongs to $\delta_{1}^{3}$; the second, to $\delta_{2}^{3}$.)

$\Gamma\left(\delta^{*}\right):(3,2) ;(2,3) ;(1,4)$. (The first entry belongs to $\delta_{2}^{*} ;$ the second, to $\delta_{1}^{*}$.)

$\Gamma(\delta):(4,1,2),(3,2,2),(2,3,2),(1,4,2) ;(3,1,3),(2,2,3),(1,3,3) ;(2,1,4)$, $(1,2,4)$. (The first entry belongs to $\delta_{4}$; the second, to $\delta_{5}$; the third, to $\delta_{7}$.)

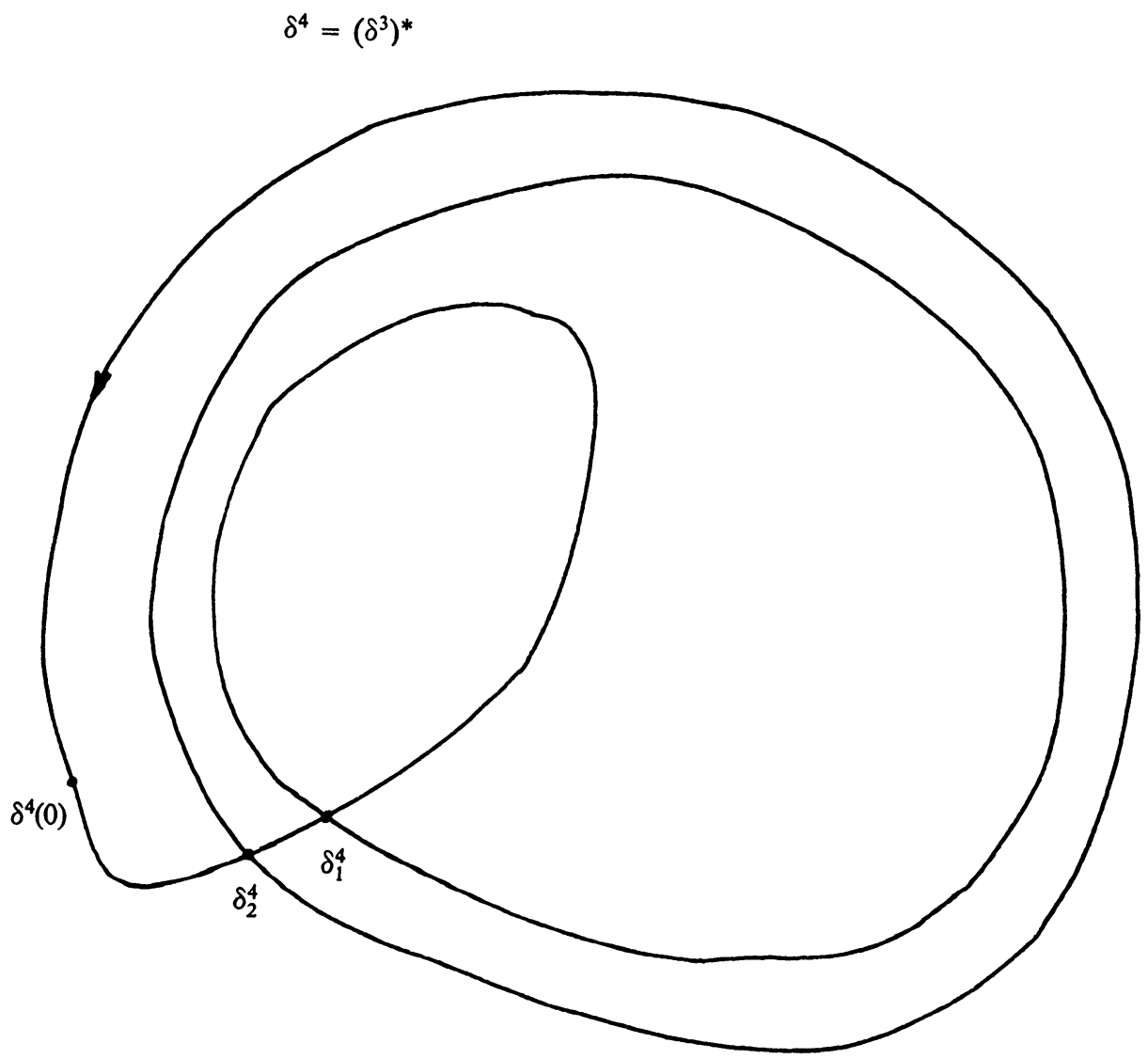

FIGURE 4 
4. The cut process. Let $\delta$ be a normal representation of a closed curve. In this paragraph we define representations which are "more like" representations with $\lambda \equiv 1$. We show that these representations determine whether $\delta$ is an interior boundary and, if so, determine the branch point structure of $\delta$.

Assume $\delta$ has nonnegative circulation; otherwise, it cannot be an interior boundary. If $\delta$ has $\lambda(j)=1$ for all vertices $\delta_{j}$, then Theorem 1 gives the desired information about $\delta$ and $\Gamma(\delta)$. So we assume that this is not the case; we select a positive outer point $\delta(0)$ and, with respect to that, number the vertices as in Paragraph 2. We select the least $q$ such that $\lambda(q)=-1$.

Suppose $P=\left\{\delta_{j} \mid j<q, t_{j}^{\prime}, t_{j}^{\prime \prime}<t_{q}^{\prime}\right\}$. By choice of $q$, for any $\delta_{i}$ and $\delta_{j}$ in $P$, either $i \supset j, j \supset i$, or $i \mid j$. A nonempty subset $P^{\prime}$ of $P$ will be called a nest if

(i) there exist some $a, b<q$ such that $\delta_{a}, \delta_{b}$ are not in $P$ and $0<t_{a}^{\prime}<t_{j}^{\prime}<t_{j}^{\prime \prime}<t_{b}^{\prime}<t_{q}^{\prime}$ for every $\delta_{j}$ in $P^{\prime}$.

(ii) given any $\delta_{i}$ and $\delta_{j}$ in $P^{\prime}$, if there exists a $\delta_{k}, k<q$, with $t_{i}^{\prime}<t_{k}^{\prime}<t_{j}^{\prime}$, then $\delta_{k}$ must be in $P^{\prime}$.

Let the nests of $\delta$, if there are any, be $P_{1}, P_{2}, \ldots, P_{p}$. Given a nest $P_{r}$, pick $s_{r}$ and $u_{r}$ such that $0<s_{r}<t_{j}^{\prime}<t_{j}^{\prime \prime}<u_{r}<t_{q}^{\prime}$ for every $\delta_{j} \in P_{r}$ and if $k$ is such that $s_{r}<t_{k}^{\prime}$ or $t_{k}^{\prime \prime}<u_{r}$, then $\delta_{k}$ is in $P_{r}$. Let $\gamma^{r}$ represent an arc which intersects [ $\delta$ ] only in its end points $\delta\left(s_{r}\right)$ and $\delta\left(u_{r}\right)$, and such that $\delta\left(s_{r}\right) \delta\left(u_{r}\right)(\delta)+\delta\left(u_{r}\right) \delta\left(s_{r}\right)\left(\gamma^{r}\right)$ describes a curve of nonnegative circulation. (See Figure 5.) Define $I=\bigcup\left\{\left(t_{j}^{\prime}, t_{j}^{\prime \prime}\right) \mid \delta_{j}\right.$ in $\left.P\right\}$. Let $\beta$ be a parametrization of the $\operatorname{arc}\left[\delta(0) \delta_{q}(\delta)\right]-\delta(I)$, oriented from $\delta(0)$ to $\delta_{q}$.

For any $m<q$ with $\delta_{m}$ not in $P$ one of the following cases must arise

$$
\begin{aligned}
& A(m): t_{m}^{\prime}<t_{q}^{\prime}<t_{q}^{\prime \prime}<t_{m}^{\prime \prime}, \\
& B(m): t_{m}^{\prime}<t_{q}^{\prime}<t_{m}^{\prime \prime}<t_{q}^{\prime \prime} .
\end{aligned}
$$

Other cases are ruled out by choice of $m$ and $q$.

Now we are ready to define the cuts of $\delta: \delta^{*}, \delta^{* *}, \varepsilon, \varepsilon^{r}, \zeta^{r}$.

Case $A(m)$. Define $\delta^{*}$ and $\delta^{* *}$ by

$$
\begin{aligned}
\delta^{*} & =\delta_{m} \delta_{q}(\beta)+\delta\left(t_{q}^{\prime \prime}\right) \delta\left(t_{m}^{\prime \prime}\right)(\delta), \\
\delta^{* *} & =\delta(0) \delta\left(t_{q}^{\prime \prime}\right)(\delta)+\delta_{q} \delta_{m}(-\beta)+\delta\left(t_{m}^{\prime \prime}\right) \delta(2 \pi)(\delta) .
\end{aligned}
$$

See Figures 6 and 7.

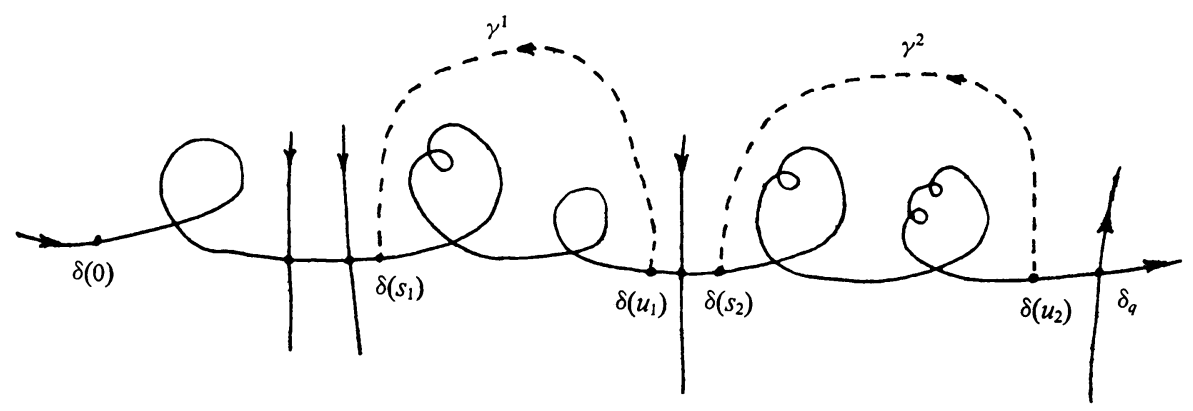

FIGURE 5 


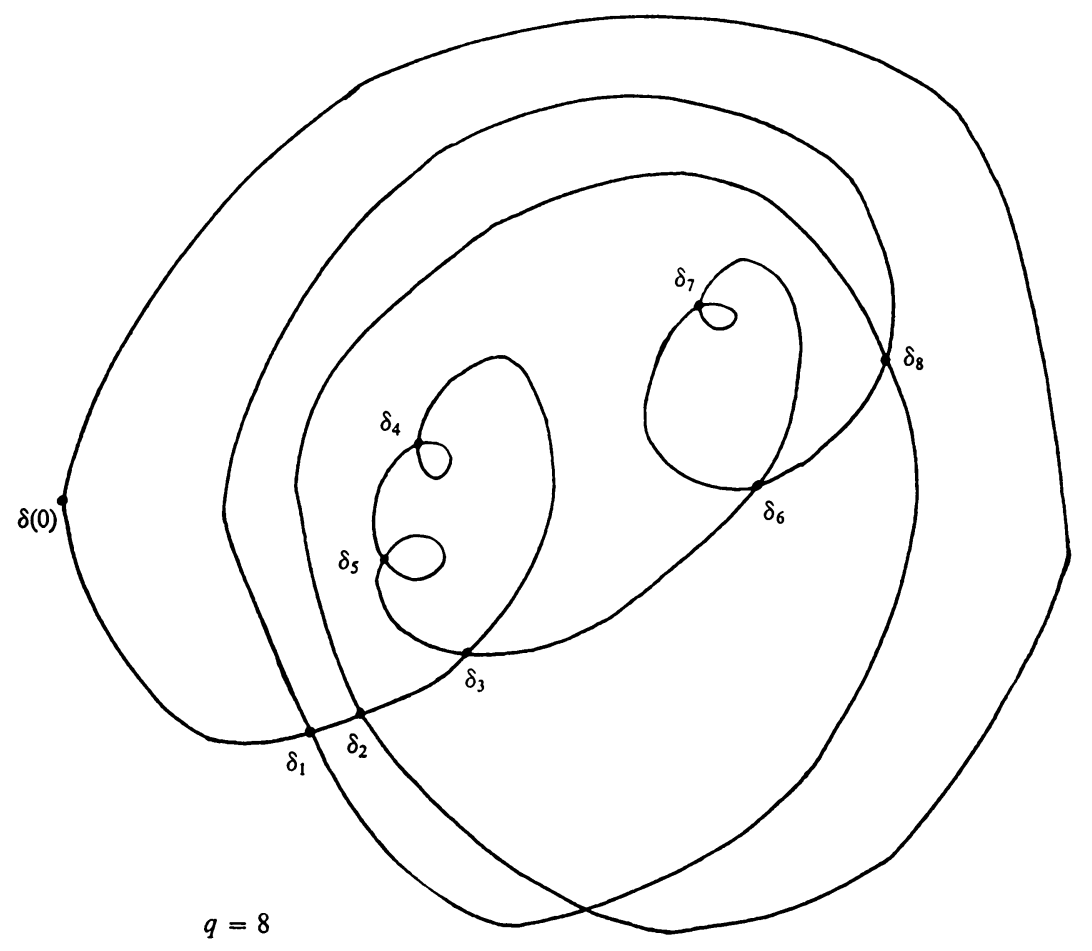

FIGURE 6

Case $B(m)$. Define $\delta^{*}$ and $\delta^{* *}$ by

$$
\begin{aligned}
\delta^{*} & =\delta_{q} \delta_{m}(-\beta)+\delta\left(t_{m}^{\prime \prime}\right) \delta\left(t_{q}^{\prime \prime}\right)(\delta) \\
\delta^{* *} & =\delta(0) \delta\left(t_{m}^{\prime \prime}\right)(\delta)+\delta_{m} \delta_{q}(\beta)+\delta\left(t_{q}^{\prime \prime}\right) \delta(2 \pi)(\delta) .
\end{aligned}
$$

See Figure 8.

Also in this case, if there are $p$ nests, then for each $r, 1 \leqq r \leqq p$, with $t_{m}^{\prime}<s_{r}$ define $\varepsilon^{r}$ and $\zeta^{r}$ by

$$
\begin{aligned}
& \varepsilon^{r}=\delta_{m} \delta\left(u_{r}\right)(\beta)+\delta\left(u_{r}\right) \delta\left(s_{r}\right)\left(\gamma^{r}\right)+\delta\left(s_{r}\right) \delta\left(u_{r}\right)(\beta)+\delta\left(u_{r}\right) \delta\left(t_{m}^{\prime \prime}\right)(\delta) \quad \text { (Figure 9), } \\
& \zeta^{r}=\delta(0) \delta\left(s_{r}\right)(\delta)+\delta\left(s_{r}\right) \delta\left(u_{r}\right)(\beta)+\delta\left(u_{r}\right) \delta\left(s_{r}\right)\left(\gamma^{r}\right)+\delta\left(s_{r}\right) \delta_{q}(\beta)+\delta\left(t_{q}^{\prime \prime}\right) \delta(2 \pi)(\delta)
\end{aligned}
$$

(Figure 10).

Let $k$ be the least positive integer such that $t_{m}^{\prime}<s_{k}$. If no such $k$ exists, let $\varepsilon=\delta^{*}$; otherwise define $\varepsilon$ by

$$
\begin{aligned}
\varepsilon= & \delta(0) \delta\left(s_{k}\right)(\delta)+\delta\left(s_{k}\right) \delta\left(u_{k}\right)\left(-\gamma^{k}\right)+\delta\left(u_{k}\right) \delta\left(s_{k+1}\right)(\delta)+\delta\left(s_{k+1}\right) \delta\left(u_{k+1}\right)\left(-\gamma^{k+1}\right) \\
& +\cdots+\delta\left(u_{p-1}\right) \delta\left(s_{p}\right)(\delta)+\delta\left(s_{p}\right) \delta\left(u_{p}\right)\left(-\gamma^{p}\right)+\delta\left(u_{p}\right) \delta(2 \pi)(\delta) .
\end{aligned}
$$

See Figure 11.

Before we begin proving theorems about these representations, we will modify $\varepsilon^{r}$ and $\zeta^{r}$ so that they are normal. Some of the other representations will be modified in $\S 5$. Let $x$ be a point on $[\beta]$ such that $x$ is encountered before $\delta\left(s_{r}\right)$ and there are no vertices of $\delta$ on [ $\beta]$ between $x$ and $\delta\left(s_{r}\right)$. Let $\alpha$ represent an arc which touches [ $\delta$ ] only at its end points, $x$ and $\delta\left(u_{r}\right)$. Assume $\alpha$ is oriented from $x$ to $\delta\left(u_{r}\right)$ and is 
Case $\mathrm{A}(2): t_{2}^{\prime}<t_{8}^{\prime}<t_{8}^{\prime \prime}<t_{2}^{\prime \prime}$
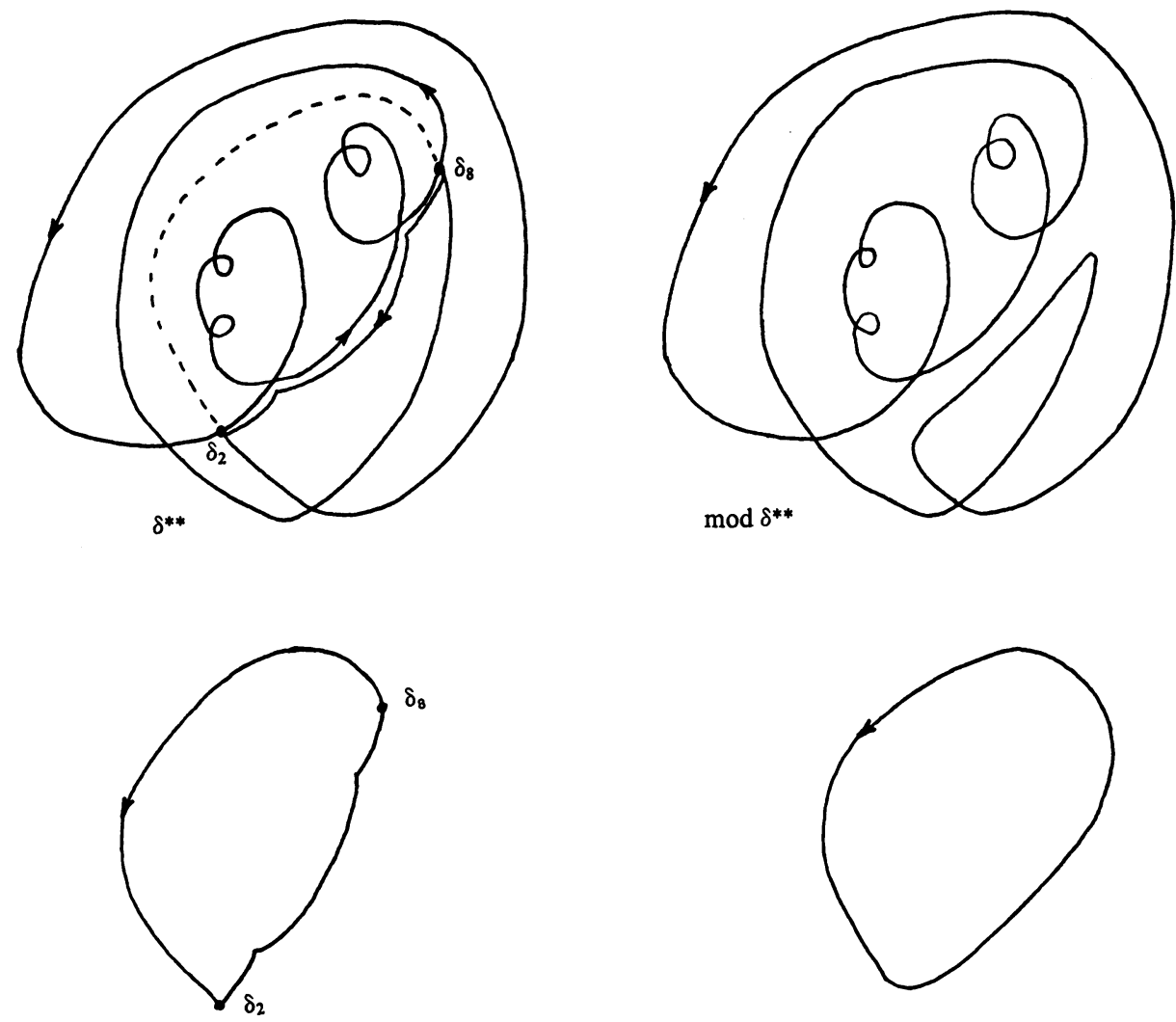

$\delta^{*}$

$\bmod \delta^{*}$

Figure 7
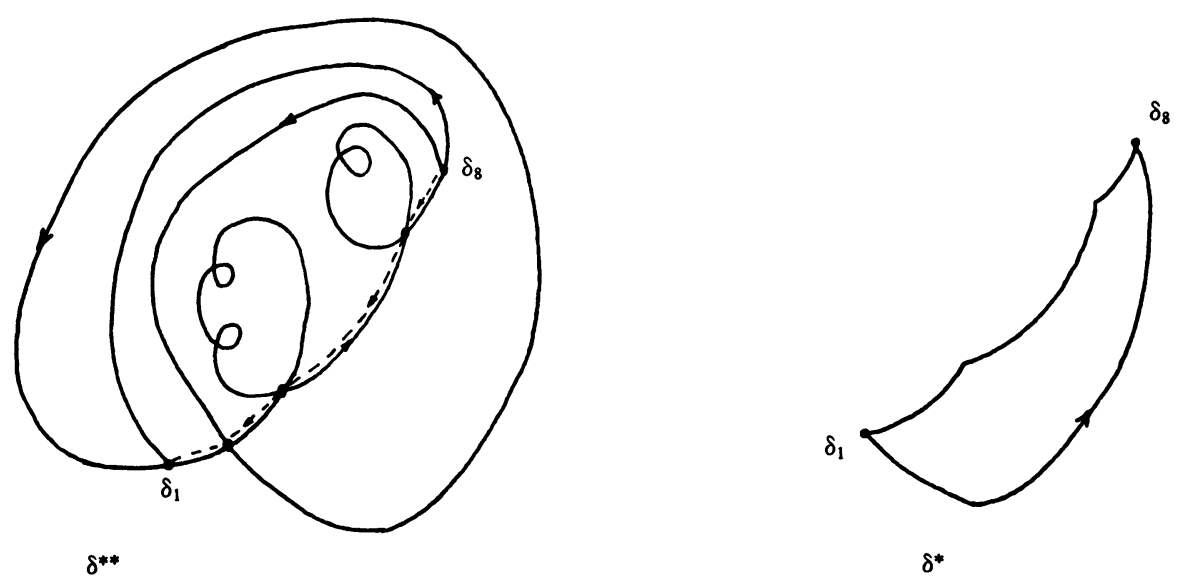

FIGURE 8 

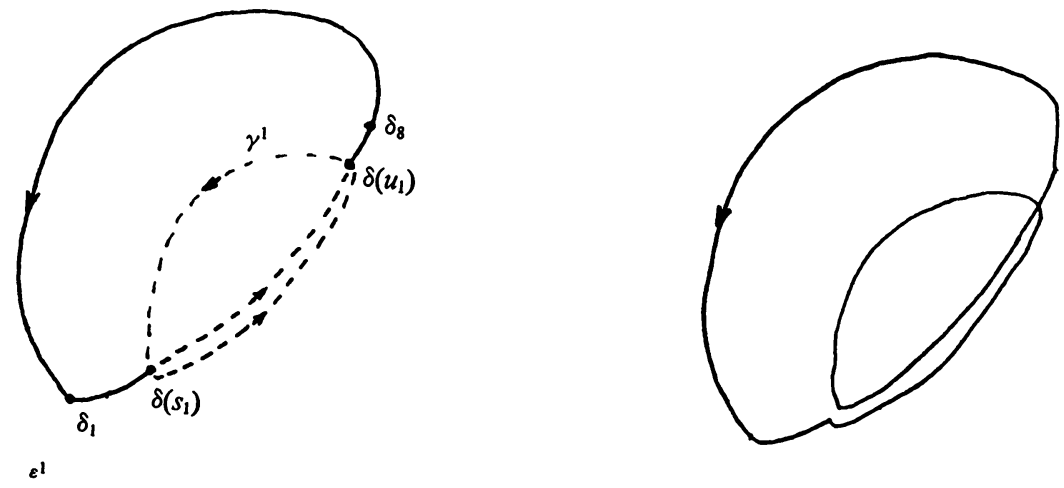

$\bmod e^{1}$

Figure 9
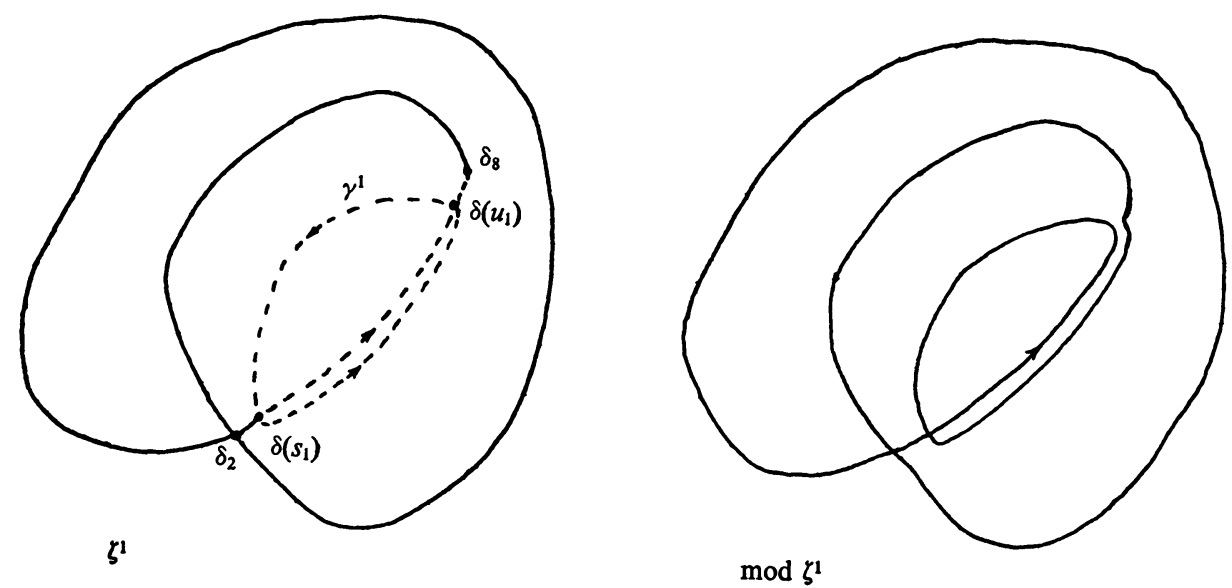

FIGURE 10
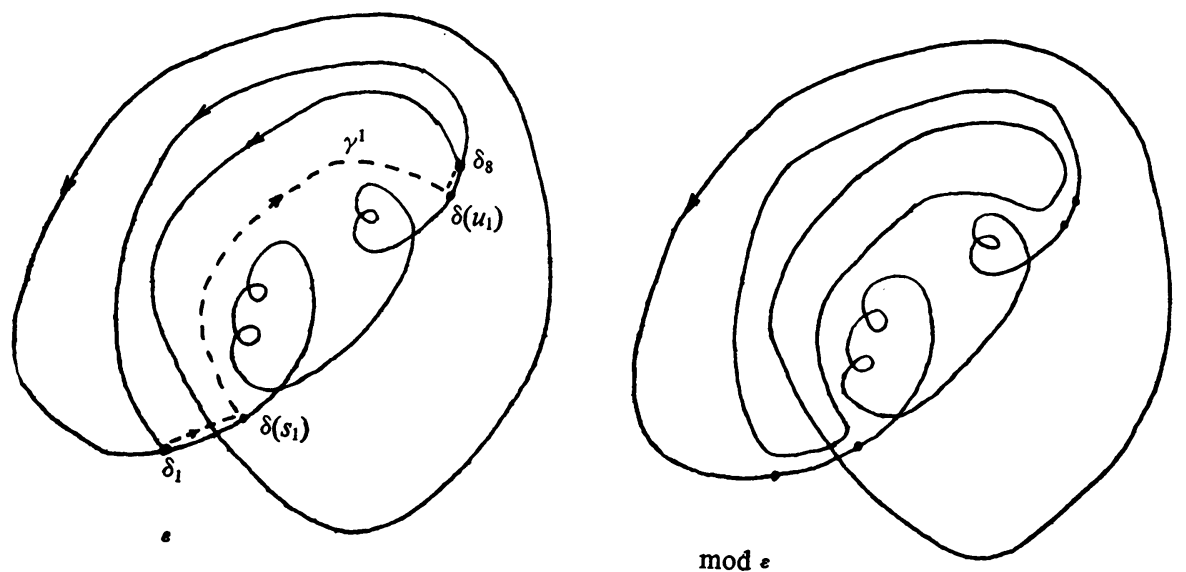

FIGURE 11 
such that $x \delta\left(u_{r}\right)(\alpha)+\delta\left(u_{r}\right) x(-\beta)$ is a positively oriented Jordan curve (i.e., $\alpha$ lies to the right of $\beta$ ). Then define $\bmod \varepsilon^{r}$ by

$$
\bmod \varepsilon^{r}=\delta_{m} x(\beta)+x \delta\left(u_{r}\right)(\alpha)+\delta\left(u_{r}\right) \delta\left(s_{r}\right)\left(\gamma^{r}\right)+\delta\left(s_{r}\right) \delta\left(u_{r}\right)(\beta)+\delta\left(u_{r}\right) \delta\left(t_{m}^{\prime \prime}\right)(\delta) .
$$

(See Figure 9.)

Similarly, let $y$ be a point of $[\beta]$ which is encountered after $\delta\left(u_{r}\right)$ and such that there are no vertices of $\delta$ between $\delta\left(u_{r}\right)$ and $y$. Let $\alpha^{*}$ represent an arc oriented from $\delta\left(s_{r}\right)$ to $y$ with $\alpha^{*}$ to the right of $\beta$. Define $\bmod \zeta^{r}$ by

$$
\begin{aligned}
\bmod \zeta^{r}= & \delta(0) \delta\left(s_{r}\right)(\delta)+\delta\left(s_{r}\right) \delta\left(u_{r}\right)(\beta)+\delta\left(u_{r}\right) \delta\left(s_{r}\right)\left(\gamma^{r}\right) \\
& +\delta\left(s_{r}\right) y\left(\alpha^{*}\right)+y \delta_{q}(\beta)+\delta\left(t_{q}^{\prime \prime}\right) \delta(2 \pi)(\delta) . \quad \text { (See Figure 10.) }
\end{aligned}
$$

Smooth $\bmod \varepsilon^{r}$ and $\bmod \zeta^{r}$ so that they are regular. We leave it to the reader to prove that mod $\varepsilon^{r}$ is normal, mod $\varepsilon^{r}$ is an interior boundary if and only if $\varepsilon^{r}$ is, and $\Gamma\left(\varepsilon^{r}\right)=\Gamma\left(\bmod \varepsilon^{r}\right)$. A similar statement is true for $\zeta^{r}$.

Hoping that the reader's patience is not exhausted, we make one more definition. Let $\eta$ be a representation with $\lambda \equiv 1$, suppose it is parametrized by the usual angle parameter $0 \leqq \theta \leqq 2 \pi$. The outer boundary of $\eta$ is a Jordan curve; suppose it has a positively oriented parametrization $\phi(\theta), 0 \leqq \theta \leqq 2 \pi$, where $\phi(0)=\eta(0)$. Define $\eta$ aug $z^{k}, k \geqq 1$, by

$$
\begin{aligned}
\left(\eta \text { aug } z^{k}\right)(\theta) & =\eta(k \theta), & 0 \leqq \theta \leqq \frac{2 \pi}{k+1} & \\
& =\phi((k+1) \theta-2 j \pi), & \frac{2 j \pi}{k+1} \leqq \theta \leqq \frac{2(j+1) \pi}{k+1}, & j=1,2, \ldots, k .
\end{aligned}
$$

Note that $\eta$ aug $z^{k}$ has the same topological properties as a representation $\eta^{*}$ with $\lambda \equiv 1$ whose chains of vertices are described as follows:

If $\delta_{i_{1}}>\delta_{i_{2}}>\cdots>\delta_{i_{r}}$ is a chain of $\eta$, then $\delta_{1}>\delta_{2}>\cdots>\delta_{k}>\delta_{i_{1}+k}>\cdots>\delta_{i_{r}+k}$ is a chain of $\eta^{*}$. (See Figure 12.) Thus, we can apply the results of $\S 3$ to $\eta$ aug $z^{k}$.
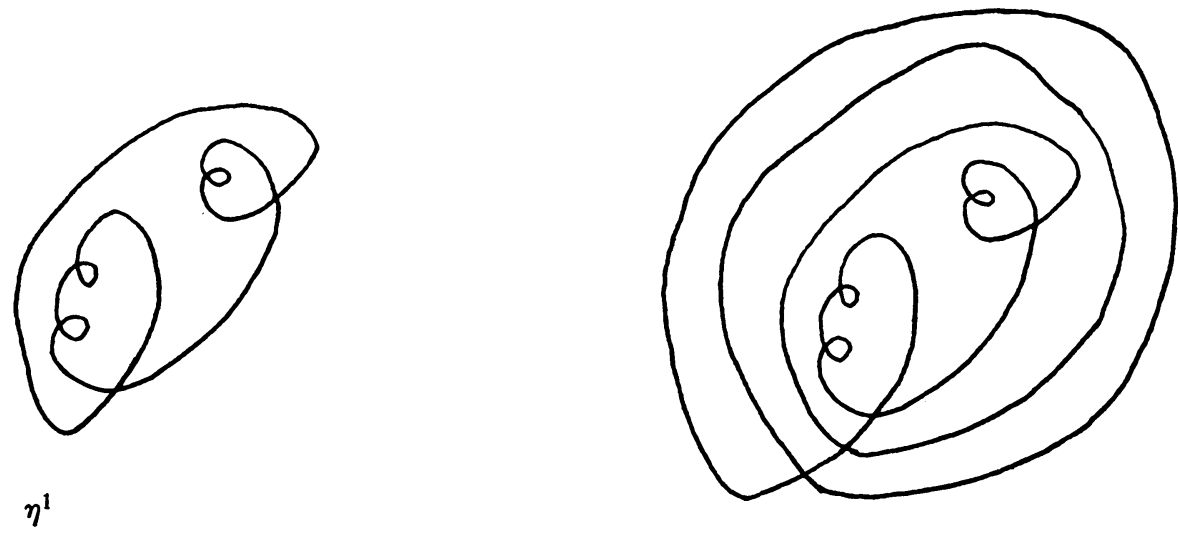

$\eta^{1}$ aug $z^{2}$ (modified) 
THEOREM 2. Suppose for some $m<q$, Case $A(m)$ or $B(m)$ holds and $\delta^{*}$ and $\delta^{* *}$ are interior boundaries. Then $\delta$ is an interior boundary. Moreover, $\Gamma(\delta) \supset\left\{\mu^{*} \cup \mu^{* *} \mid \mu^{*}\right.$ is in $\Gamma\left(\delta^{*}\right)$ and $\mu^{* *}$ is in $\left.\Gamma\left(\delta^{* *}\right)\right\}$.

Proof. The proof follows along the lines of the proofs of Theorem $7^{\prime}$ and Theorem 7" [4, p. 56].

THEOREM 3. If for some $m<q$, Case $B(m)$ holds and $\delta^{*}$ and $\varepsilon$ are interior boundaries, then $\delta$ is an interior boundary. Moreover, $\Gamma(\delta) \supset\left\{\mu \cup \nu \mid \mu\right.$ is in $\Gamma\left(\delta^{*}\right)$ and $\nu$ is in $\left.\Gamma(\varepsilon)\right\}$.

Proof. Observe that if $\varepsilon$ is an interior boundary with some properly interior extension $g$, then $\delta^{* *}$ (as defined in Case $B(m)$ ) is an interior boundary which has an extension with the same branch points as $g$. Then the proof again is similar to the proof of Theorem $7^{\prime}[4$, p. 55].

THEOREM 4. If for some $m<q$ and some $r, 1 \leqq r \leqq p$ and $t_{m}^{\prime}<s_{r}$, it is the case that $\varepsilon^{r}$ and $\zeta^{r}$ are interior boundaries, then $\delta^{* *}$ is an interior boundary. Suppose $\mu$ is in $\Gamma\left(\bmod \varepsilon^{r}\right)$ with $|\mu|=k^{*}$ where $\mu(1), \ldots, \mu(k)$ belong to the terminal vertex $\delta\left(u_{r}\right)$ of $\varepsilon^{r}$. Define $M=\sum_{i=1}^{k} \mu(i)$ and $\mu^{*}=\mu(k+1), \ldots, \mu\left(k^{*}\right)$. (Let $\mu^{*}=\Phi$ if $k=k^{*}=1$.) Similarly let $\nu$ be in $\Gamma\left(\bmod \zeta^{r}\right)$ with $|\nu|=L^{*}$ and suppose $\nu(1), \ldots, \nu(L)$ belong to the terminal vertex $\delta\left(s_{r}\right)$ of $\zeta^{r}$. Define $N=\sum_{i=1}^{L} \nu(i)$ and $\nu^{*}=\nu(L+1), \ldots, \nu\left(L^{*}\right)$. (Let $\nu^{*}=\Phi$ if $L=L^{*}=1$.) Let $\eta=\delta\left(s_{r}\right) \delta\left(u_{r}\right)(\delta)+\delta\left(u_{r}\right) \delta\left(s_{r}\right)\left(\gamma^{r}\right)$. If $\xi$ is in $\Gamma\left(\eta\right.$ aug $\left.z^{M+N-1}\right)$, then $\mu^{*} \cup \nu^{*} \cup \xi$ is in $\Gamma\left(\delta^{* *}\right)$.

Proof. Suppose $g_{1}$ is a properly interior extension of $\zeta^{r}$ to the Jordan domain $\bar{D}_{1}$; similarly let $g_{2}$ be a properly interior extension of $\varepsilon^{r}$ to $\bar{D}_{2}$. Let $s$ and $s^{\prime}$ be the pre-images of $\delta\left(s_{r}\right)$ under $g_{1}$; let $u$ and $u^{\prime}$ be the pre-images of $\delta\left(u_{r}\right)$ under $g_{2}$. Let the arcs $T_{1}$ in $\bar{D}_{1}$ and $T_{2}$ in $\bar{D}_{2}$ be as in Lemma 3 .

There is a point $y^{*}$ in $\bar{D}_{1}-D_{1}$ such that $g_{1}\left(y^{*}\right)=y$. Suppose $s$ is encountered before $s^{\prime}$ as $\bar{D}_{1}-D_{1}$ is traversed from $y^{*}$ to $y^{*}$ in the positive orientation. We may assume there are no branch points of $g_{1}$ mapping into Ins $\left[\delta\left(s_{r}\right) y\left(\alpha^{*}\right)+y \delta\left(s_{r}\right)(-\beta)\right]$. This fact and an application of Theorem 1 [1, p. 49] produce an arc $H$ in $\bar{D}_{1}$ mapping homeomorphically onto $\left[\delta\left(s_{r}\right) y(\beta)\right]$ where $H$ intersects $\bar{D}_{1}-D_{1}$ only at its end points $s^{\prime}$ and $y^{*}$. Let $B_{1}$ be the open disk bounded by $H, T_{1}$, and $\left[y^{*}, s\right]$.

Let $x^{*}$ be the point of $\bar{D}_{2}-D_{2}$ such that $g_{2}\left(x^{*}\right)=s$. Suppose $u$ and $u^{\prime}$ are such that $x^{*}$ is not in $\left[u, u^{\prime}\right]$. Let $z$ be the pre-image of $\delta\left(s_{r}\right)$ first encountered as $T_{2}$ is traced from $u$ to $u^{\prime}$. An argument similar to that of the previous paragraph produces an arc $K$ in $\bar{D}_{2}$ mapping homeomorphically onto $\left[x \delta\left(s_{r}\right)(\beta)\right]$, where $K$ intersects $\bar{D}_{2}-D_{2}$ only in its end points $x^{*}$ and $z$. Let $B_{2}$ be the open disk bounded by $K,\left[u^{\prime}, x^{*}\right]$, and $T_{2}^{\prime}$, the closure of the component of $T_{2}-\{z\}$ containing $u^{\prime}$.

Assume without loss of generality that $\bar{B}_{1}$ and $\bar{B}_{2}$ intersect in one and only one point, $s^{\prime}$ (on $\left.\bar{B}_{1}\right)=z$ (on $\bar{B}_{2}$ ). Let $L$ be an arc joining $s$ and $u^{\prime}$ which touches $\bar{B}_{1} \cup \bar{B}_{2}$ only in those points. Suppose $\phi$ represents $\delta\left(s_{r}\right) \delta\left(u_{r}\right)(\delta)$ on $L$. Then the representation $\phi^{*}$ defined on $T_{1} \cup T_{2}^{\prime} \cup L$ by $\phi^{*}\left|T_{1}=g_{1}, \phi^{*}\right| T_{2}^{\prime}=g_{2}, \phi^{*} \mid L=\phi$, is equivalent to a representation of $\eta$ aug $z^{M+N-1}$. This follows since $g_{1} \mid T_{1}$ is topologically $z^{M}$; 
$g_{2} \mid T_{2}$ is topologically $z^{N}$. However, one traversing of the loop by $g_{2}$ is lost due to using $T_{2}^{\prime}$ instead of $T_{2}$ and taking part of $T_{2}^{\prime}$ together with $L$ to get a representation of $\eta$.

We know that there is a properly interior extension $g_{3}$ of $\phi^{*}$ to the disk $B_{3}$ bounded by $T_{1} \cup T_{2}^{\prime} \cup L$. If we define $f$ on $\bar{B}_{1} \cup \bar{B}_{2} \cup \bar{B}_{3}$ by $f \mid \bar{B}_{i}=g_{i}, 1 \leqq i \leqq 3$, then by Theorem $9[5, \mathrm{p} .336] f$ is properly interior and extends $\delta^{* *}$. Clearly, by its construction $f$ will have branch point structure as described in the theorem.

Having seen that we can construct elements of $\Gamma(\delta)$ from the cuts of $\delta$, we now show that every member of $\Gamma(\delta)$ arises in this way.

THEOREM 5. Suppose $\delta$ is an interior boundary where $\lambda \not \equiv 1$ and let $q$ be the least integer such that $\lambda(q)=-1$. Then for some $m<q$, Case $A(m)$ or Case $B(m)$ holds and $\delta^{*}$ and $\delta^{* *}$ as defined in that case are interior boundaries. If $\mu$ is in $\Gamma(\delta)$, then there exist $\mu^{*}$ in $\Gamma\left(\delta^{*}\right)$ and $\mu^{* *}$ in $\Gamma\left(\delta^{* *}\right)$ such that $\mu=\mu^{*} \cup \mu^{* *}$.

Proof. Suppose $f$ is a properly interior extension of $\delta$ to $\bar{D}$. Since $\lambda(q)=-1$, by Theorem 1 [1, p. 49], there is an $\operatorname{arc} A$ in $\bar{D}$ with one end point at $t_{q}^{\prime \prime}$ such that $A$ intersects $\bar{D}-D$ only at $t_{q}^{\prime \prime}$ and possibly its other end point $b$; also, $f(A) \subset[\beta]$. If $b$ is in $D$, this same Theorem 1 gives that $f(b)=\beta(0)=\delta(0)$. However, an interior mapping cannot map a point of $D$ into the boundary of $f(D)$, but $\delta(0)$ is an outer point and hence, on the boundary on $f(D)$. We can conclude that $b$ is in $\bar{D}-D$. Since $f$ is a local homeomorphism at each point of $\bar{D}-D$, it must be that $b=t_{m}^{\prime \prime}$ for some $m$ and since $f(b)$ is in $[\beta], m<q$. As we have remarked before, by choice of $m$ and $q$, either case $A(m)$ or $B(m)$ arises. Let $D_{1}$ and $D_{2}$ be the components of $D-A$, where $\bar{D}_{2}-D_{2}$ contains $f^{-1}(\delta(0))$. By definition, $f \mid \bar{D}_{1}-D_{1}=\delta^{*}$ and $f \mid \bar{D}_{2}-D_{2}=\delta^{* *}$; thus, $\delta^{*}$ and $\delta^{* *}$ are interior boundaries.

No branch point of $f$ can lie on $A$ since $f(A) \subset[\beta] \subset[\delta]$ and we are assuming no branch points map onto [8]. Hence, the branch points of $f$ are in $D_{1} \cup D_{2}$. Let $\mu^{*}=\mu\left(j_{1}\right), \ldots, \mu\left(j_{k}\right)$ be the multiplicities of the branch points of $f$ in $D_{1}$ and $\mu^{* *}=\mu\left(j_{k+1}\right), \ldots, \mu\left(j_{s}\right)$, those in $D_{2}$. Clearly $\mu^{*}$ is in $\Gamma\left(\delta^{*}\right), \mu^{* *}$ is in $\Gamma\left(\delta^{* *}\right)$, and $\mu^{*} \cup \mu^{* *}$ comprises all the multiplicities of the branch points of $f$.

THEOREM 6. Suppose $\delta^{* *}$ is an interior boundary and $q$ is the least integer such that $\lambda(q)=-1$. Suppose for some $m<q$, Case $B(m)$ holds and for some $r, 1 \leqq r \leqq p$, with $t_{m}^{\prime}<s_{r}, \varepsilon^{r}$ and $\zeta^{r}$ are interior boundaries. If $\rho$ is in $\Gamma\left(\delta^{* *}\right)$, then there exist $\mu$ in $\Gamma\left(\varepsilon^{r}\right)$, $\nu$ in $\Gamma\left(\zeta^{r}\right)$, and $\xi$ in $\Gamma\left(\eta\right.$ aug $\left.z^{M+N-1}\right)$ such that $\rho=\mu^{*} \cup \nu^{*} \cup \xi$, where $M, \mu^{*}, N$, $\nu^{*}$, and $\eta$ are as in Theorem 4. If for each $r, 1 \leqq r \leqq p$, with $t_{m}^{\prime}<s_{r}$, either $\varepsilon^{r}$ or $\zeta^{r}$ fails to be an interior boundary, then $\varepsilon$ is an interior boundary. In this case $\Gamma\left(\delta^{* *}\right)=\Gamma(\varepsilon)$.

Proof. Let $f^{* *}$ be an extension of $\delta^{* *}$ to $\bar{D}$. Suppose $\alpha^{j}$ describes an arc which intersects [ $\delta]$ only at its end points $\delta\left(s_{j}\right)$ and $\delta\left(u_{j}\right), 1 \leqq j \leqq p$; let it be oriented from $\delta\left(u_{j}\right)$ to $\delta\left(s_{j}\right)$. If $K_{j}$ is the Jordan curve $\left[\delta\left(s_{j}\right) \delta\left(u_{j}\right)(\beta)+\delta\left(u_{j}\right) \delta\left(s_{j}\right)\left(\alpha^{j}\right)\right]$, then $\alpha^{j}$ should be located so that $\left[\gamma^{j}\right] \subset K_{j} \cup$ Ins $K_{j}$. Let $A$ be the arc on $\bar{D}-D$ such that $f^{* *} \mid A$ describes $\delta \mid\left[t_{m}^{\prime}, t_{q}^{\prime}\right]$ and let $B$ be the arc such that $\delta \mid B$ describes $\delta_{m} \delta_{q}(\beta)$. Suppose $x_{j}^{\prime}$ 
and $y_{j}^{\prime}$ are in $A$ and $x_{j}$ and $y_{j}$ are in $B$, where $f^{* *}\left(x_{j}\right)=f^{* *}\left(x_{j}^{\prime}\right)=\delta\left(s_{j}\right)$ and $f^{* *}\left(y_{j}\right)=$ $f^{* *}\left(y_{j}\right)=\delta\left(u_{j}\right)$.

By an argument similar to that in Lemma 3 , there is an arc $H_{j}$ in $\bar{D}$ with one end point at $x_{j}$, the other, $z_{j}$, in $A \cup B$, and $f^{* *}\left(H_{j}\right) \subset K_{j}$. Let us first suppose that for each $j, 1 \leqq j \leqq p$, the other end point $z_{j}$ is in $B$. Then since $f^{* *}$ is properly interior, $z_{j}=y_{j}$. Denote by $L_{j}$ the Jordan curve formed by $H_{j}$ and $H_{j}^{\prime}=\left[x_{j}, y_{j}\right]$. By Theorem $4.2\left[6\right.$, p. 96], $f^{* *} \mid L_{j}$ is topologically equivalent to the mapping $w=z^{k}$ for some positive integer $k$. Hence, there exists a properly interior mapping $g_{j}$ on $L_{j} \cup \operatorname{Ins} L_{j}$ such that $g_{j} \mid H_{j}=f^{* *}$ and $g_{j} \mid H_{j}^{\prime}$ traces $-\gamma^{j}$. Also, $g_{j}$ can be taken to have the same branch point structure on $L_{j} \cup$ Ins $L_{j}$ as $f^{* *}$ does. If we define $h$ to be $f^{* *}$ on $\bar{D}-\bigcup_{j=1}^{p}\left(L_{j} \cup \operatorname{Ins} L_{j}\right)$ and $g_{j}$ on $L_{j} \cup \operatorname{Ins} L_{j}, 1 \leqq j \leqq p$, then by Theorem 9 [5, p. 336], $h$ is properly interior on $\bar{D}$; by definition of $\varepsilon, h$ extends $\varepsilon$. Since $h$ has the same branch point structure as $f^{* *}$, it must be that every $\mu$ in $\Gamma\left(\delta^{* *}\right)$ which has an extension $f^{* *}$ with every $z_{j}$ in $B$ is in $\Gamma(\varepsilon)$. We shall soon prove that if some $z_{j}$ is in $A$, then $\varepsilon^{j}$ and $\zeta^{j}$ are interior boundaries. Hence, in the case that $\varepsilon^{j}$ and $\zeta^{j}$, $1 \leqq j \leqq p$, fail to be interior boundaries, every extension $f^{* *}$ of $\delta^{* *}$ is as above. We conclude that in this case, $\Gamma\left(\delta^{* *}\right) \subset \Gamma(\varepsilon)$.

Now we show that $\Gamma(\varepsilon) \subset \Gamma\left(\delta^{* *}\right)$. Let $g$ be a properly interior extension of $\varepsilon$ to $\bar{D}$. Let $B_{j}=\left[u_{j}, v_{j}\right]$ be the arc of $\bar{D}-D$ mapping onto $\gamma^{j}, 1 \leqq j \leqq p$. Choose $C_{j}$, an arc in $E^{2}-D$, so that $C_{j}$ intersects $\bar{D}-D$ only at its end points $u_{j}$ and $v_{j}$ and so that traversing $B_{j}$ from $u_{j}$ to $v_{j}$ and then $C_{j}$ from $u_{j}$ to $v_{j}$ is in the negative orientation. Let $g_{j}$ be a homeomorphism such that $g_{j} \mid B_{j}=g$ and $g_{j}$ maps $C_{j}$ onto $\left[\delta\left(s_{j}\right) \delta\left(u_{j}\right)(\beta)\right]$. Clearly $g_{j}$ can be extended to be a homeomorphism on $E_{j}=B_{j} \cup C_{j} \cup \operatorname{Ins}\left(B_{j} \cup C_{j}\right)$. Call this extended map $g_{j}^{*}$. If we define $h$ on $E=\bigcup_{j=1}^{p} E_{j} \cup \bar{D}$ by $h \mid \bar{D}=g$ and $h \mid \bar{E}_{j}=g_{j}^{*}$, then $h$ is a properly interior extension of $\delta^{* *}$ with the same branch point structure as $g$. Hence, $\Gamma(\varepsilon) \subset \Gamma\left(\delta^{* *}\right)$ and so $\Gamma(\varepsilon)=\Gamma\left(\delta^{* *}\right)$.

Now suppose for some $r, 1 \leqq r \leqq p$, that $z_{r}$ is in $A$. Since $f^{* *}$ is properly interior, it must be that $z_{r}=y_{r}^{\prime}$. A similar argument to that used for $H_{r}$ produces an arc $H_{r}^{\prime \prime}$ in $\bar{D}$ such that $y_{r}$ is one end point, $x_{r}^{\prime}$ is the other, $H_{r}^{\prime \prime} \cap(\bar{D}-D)=\left\{x_{r}^{\prime}, y_{r}\right\}$, and $f^{* *}\left(H_{r}^{\prime \prime}\right) \subset K_{r}$. Let $D_{2}$ be the Jordan domain bounded by $H_{r}, H_{r}^{\prime \prime}, H_{r}^{\prime}$, and $\left[x_{j}^{\prime}, y_{j}^{\prime}\right]$. Let $D_{1}$ be the component of $D-\bar{D}_{2}$ with the pre-image under $f^{* *}$ of $\delta(0)$ on its boundary; $D_{3}$, the other component. (We note in passing that for the given $f^{* *}$, there is no $H_{j}, j \neq r$, with one end point in $A$ and the other in $B$. This follows from the fact that one end point of $H_{j}$ would be in $D_{1}$, the other in $D_{3}$. This is impossible since $H_{j} \cap H_{r}=\varnothing$.) Let $P$ be an arc in $\bar{D}_{2}$ which intersects $\bar{D}_{1}$ only at its end points $x_{r}^{\prime}$ and $y_{r}$. Suppose that $g$ is a function defined on $P$ which represents $\delta\left(s_{r}\right) \delta\left(u_{r}\right)(\beta)+$ $\delta\left(u_{r}\right) \delta\left(s_{r}\right)\left(\gamma^{r}\right)+\delta\left(s_{r}\right) \delta\left(u_{r}\right)(\beta)$. If $N$ is the number of pre-images of $\delta\left(s_{r}\right)$ on $H_{r}^{\prime \prime}$, then the function $g^{*}$ on the Jordan curve $P \cup H_{r}^{\prime \prime}$ defined by $g^{*}\left|P=g, g^{*}\right| H_{r}^{\prime \prime}=f^{* *}$, is topologically equivalent to $w=z^{N+1}$. Extend $g^{*}$ to be properly interior on $E_{1}=P$ $\cup H_{r}^{\prime \prime} \cup \operatorname{Ins}\left(P \cup H_{r}^{\prime \prime}\right)$. Defining $g^{* *}$ on $\bar{D}_{1} \cup E_{1}$ by $g^{* *}\left|\bar{D}_{1}=f^{* *}, g^{* *}\right| E_{1}=g^{*}$, we obtain a properly interior extension of $\zeta^{r}$.

Again we can argue that there are no branch points of $f^{* *}$ on $\bar{D}_{i}-D_{i}$ for $i=1,2,3$. 
Let $\nu^{* *}=\nu(1), \ldots, \nu(L)$ denote the multiplicities of the branch points of $g^{*}$ and let $\nu^{*}=\nu(L+1), \ldots, \nu\left(L^{*}\right)$ be the multiplicities of the branch points of $f^{* *}$ in $D_{1}$. Note that $\nu=\nu^{* *} \cup \nu^{*}$ is in $\Gamma\left(\zeta^{r}\right)$ and $\sum_{i=1}^{L} \nu(i)=N$.

By a similar argument we can produce a properly interior extension of $\varepsilon^{r}$ with $\mu$ in $\Gamma\left(\varepsilon^{r}\right)$ such that $\mu^{*}=\mu(k+1), \ldots, \mu\left(k^{*}\right)$ are the multiplicities of the branch points of $f^{* *}$ in $D_{3}$ and $\sum_{i=1}^{k} \mu(i)=M$, where $M$ is the number of pre-images of $\delta\left(u_{r}\right)$ on $H_{r}$. We now only have to note that by definition of $M$ and $N, f^{* *} \mid \bar{D}_{2}-D_{2}$ represents $\eta \bmod z^{M+N-1}$ where $\eta$ is as in Theorem 4. Letting $\xi$ be the sequence of multiplicities of branch points of $f^{* *}$ in $D_{2}$, we have that $\xi$ is in $\Gamma\left(\eta\right.$ aug $\left.z^{M+N-1}\right)$ and the branch point multiplicities of $f^{* *}$ are $\mu^{*} \cup \nu^{*} \cup \xi$. The theorem is thus proved.

5. The finiteness of the algorithm. It is now necessary to show that we are in some sense better off by considering the cuts instead of the original representation $\delta$. We have already observed that the branch point structure of $\delta$ is reflected by that of its cuts. Now we modify the cuts so that they are normal and prove that they have strictly less vertices than $\delta$. Thus, after a finite number of steps, the cut process will terminate, leaving normal representations which either have $\lambda \equiv 1$ or are Jordan curves. In either case, the branch point structure is determined; in the former case, by Theorem 1 .

In Case $A(m)$, define $\bmod \delta^{*}$ and $\bmod \delta^{* *}$ as in case $\mathrm{II}^{\prime}(\mathrm{j})$ [4, p. 57]. (See Figure 7.) In Case $B(m)$, when there are no nests of $\delta$ with respect to $q$, define mod $\delta^{*}$ and $\bmod \delta^{* *}$ as in case $\mathrm{II}^{\prime \prime}(\mathrm{j})[4, \mathrm{p} .58]$. Also, in Case $B(m)$, when there are nests, $\varepsilon$ is modified as in case II" $(\mathrm{j})$. (See Figure 11.) It is not difficult to show $\delta^{*}$ is an interior boundary if and only if $\bmod \delta^{*}$; also, $\Gamma\left(\delta^{*}\right)=\Gamma\left(\bmod \delta^{*}\right)$. A similar statement holds for $\bmod \delta^{* *}$ and $\bmod \varepsilon$. By Theorem $10\left[4\right.$, p. 59] we have that $\bmod \delta^{*}$, $\bmod \delta^{* *}, \bmod \varepsilon,($ in the appropriate cases) have $\leqq n-2$ vertices, where $n$ is the number of vertices of $\delta$.

If there are no nests of $\delta$ with respect to $\delta_{q}$, then we use $\bmod \delta^{*}$ and $\bmod \delta^{* *}$ as the cuts. When there are nests, the modification process of [4] would cause $\delta^{* *}$ to have more vertices than $\delta$; hence the introduction of the cuts $\varepsilon^{r}$ and $\zeta^{r}$. We have already defined $\bmod \varepsilon^{r}$ and $\bmod \zeta^{r}$ and observed that they properly preserve critical point structure. Finally, we show that they have less vertices than $\delta$.

Theorem 7. In Case $B(m), \bmod \varepsilon^{r}$ and $\bmod \zeta^{r}$ have $\leqq n-2$ vertices, where $\delta$ has $n$ vertices.

Proof. The proof will be for $\varepsilon^{r}$; that for $\zeta^{r}$ is similar.

Except for the loop added at $\delta\left(u_{r}\right), \bmod \varepsilon^{r}$ traces a subset of [ $\left.\delta\right]$ and no arc is retraced. The loop at $\delta\left(u_{r}\right)$ adds one vertex, but it replaces a nest, which had at least one vertex. These facts imply that $\bmod \varepsilon^{r}$ has $\leqq n$ vertices. However, $\delta_{m}$ and $\delta_{q}$ are not vertices of $\bmod \varepsilon^{r}$ and so the number of vertices of $\bmod \varepsilon^{r}$ is $\leqq n-2$.

A given representation may have many different cut sequences, each giving rise to a different subset of $\Gamma(\delta)$. We illustrate this by an example. 
Consider the representation $\delta$ in Figure 6 . The cuts which yield interior boundaries are shown in Figures 7-12. Notice that any $\mu$ in $\Gamma(\delta)$ which arises from a cut other than the $\varepsilon, \zeta$ cuts in Case $\mathrm{B}(1)$ has $|\mu| \geqq 4$. In Case $\mathrm{B}(1), \varepsilon^{1}$ and $\zeta^{1}$ are interior boundaries (see Figures 8 and 9). If $\mu$ is in $\Gamma\left(\varepsilon^{1}\right)$ with $|\mu|$ minimal, then $|\mu|=1$. Thus, $\mu^{*}=\Phi$ and $M=1$. Similarly for $\nu$ in $\Gamma\left(\zeta^{r}\right)$ with $|\nu|$ minimal, $\nu^{*}=\Phi$ and $N=2$. Let $\xi$ be in $\Gamma\left(\eta^{1}\right.$ aug $\left.z^{2}\right)$ (see Figure 12) with $|\xi|$ minimal. Then by Theorem 1, $|\xi|=3$. By Theorem 4, $\xi=\xi \cup \Phi \cup \Phi=\xi \cup \mu^{*} \cup \nu^{*}$ is in $\Gamma\left(\delta^{* *}\right)=\Gamma(\delta)$. It follows from Theorem 6 that any $\xi$ in $\Gamma(\delta)$ with $|\xi|$ minimal must have $|\xi| \geqq 3$. We conclude that the minimal number of branch points for any light open extension of $\delta$ is 3. This occurs only in Case $\mathrm{B}(1)$. Every $\xi$ in $\Gamma(\delta)$ with $|\xi|=3$ is in $\Gamma\left(\eta\right.$ aug $\left.z^{2}\right)$. These are listed in the example at the end of $\S 3$.

\section{REFERENCES}

1. M. L. Marx, Normal curves arising from light open mappings of the annulus, Trans. Amer. Math. Soc. 120 (1965), 45-56.

2. M. Morse, Topological methods in the theory of functions of a complex variable, Annals of Mathematics Studies No. 15, Princeton Univ. Press, Princeton, N. J., 1947.

3. C. J. Titus, $A$ theory of normal curves and some applications, Pacific J. Math. 10 (1960), 1083-1096.

4. - The combinatorial topology of analytic functions on the boundary of a disc, Acta Math. 106 (1961), 45-64.

5. C. J. Titus and G. S. Young, The extension of interiority, with some applications, Trans. Amer. Math. Soc. 103 (1962), 329-340.

6. G. T. Whyburn, Topological analysis, Princeton Univ. Press, Princeton, N. J., 1958.

VANDERBILT UNIVERSITY, NAshville, Tennessee 